\title{
Energy distribution in impulsively excited structures
}

\author{
A. Carcaterra* and N. Roveri \\ Department of Mechanics and Aeronautics, University of Rome, Rome, Italy
}

\begin{abstract}
This paper presents a novel approach to the study of shock in elastic structures. An energy storage property for linear elastic non-dissipative structures is demonstrated, then extrapolated also to damped systems. This property relates locations of energy accumulation along the structure to the form of its stiffness and mass matrices. This result can be profitable used at a design stage of complex structures undergoing shock excitations. On the theoretical ground, the obtained results disclose indeed general properties of energy distribution in dynamical systems with implications on energy localization as well as on energy equipartitioning. Finally, these findings open a new point of view on energy transmission in structures, with potential application in the context of Statistical Energy Analysis.
\end{abstract}

Keywords: Shock response, energy flow analysis, localization, equipartitioning, Statistical Energy Analysis

\section{Introduction}

Energy distribution property exhibited by complex structures is a theoretical as well as a practical engineering problem. In the present paper, a general property of energy storage for a certain class of dynamical system is demonstrated. This property is shown to have a direct application to the energy path control in shock excited structures.

In many contexts, the problem of energy distribution in complex systems is considered under two hypotheses: (i) the energy is studied considering its sharing among sub-systems, each subsystem collecting a large number of degrees of freedom, (ii) the power release is of a continuous type, meaning a finite power injection is considered.

In the present paper, we indeed study the energy storage under different hypotheses: (i) the detailed energy distribution among all the individual degrees of freedom of the system is considered, (ii) a finite energy injection is assumed, (iii) the analysis is confined to the case of linear systems. The properties derived under the previous conditions are of general interest. In fact, the energy distribution among the degrees of freedom can be directly determined without using an explicit solution of the equations of the motion, but just examining a simple expression in terms of the stiffness and mass matrices.

The present results desire to provide an additional contribution to an energy theory of structures for linear elastic systems, of interest in the context of engineering vibration analysis.

The idea has some origins in Statistical Energy Analysis (SEA) [1], that historically had the merit to make systematic the attempt to translate the point of view of statistical mechanics to engineering vibrating systems. However, this operation, partially successful, met several difficulties. Part of them are even those intrinsically related to Thermodynamics that starting from its original experimental/empiric formulation, met many problems to be connected to a microscopic modeling of matter [2]. Therefore, it is natural to expect the backflow process that starts from a dynamic particle formulation, typical of structural engineering problems, and would approach to an average global formulation in terms of energy flows, is an ambitious as well as difficult goal.

*Corresponding author: A. Carcaterra, Department of Mechanics and Aeronautics, University of Rome, 'La Sapienza', Via Eudossiana, 18, 00184, Rome, Italy. E-mail: a.carcaterra@dma.ing.uniroma1.it. 
However, in this frame, besides the large number of technical engineering papers produced in the last four decades on applications of SEA, a continuous effort has been produced in the attempt to make more robust the theoretical foundation of a thermodynamic theory for dynamical systems. Among them, the contributions [3-5] are directly addressed to a rationalization of SEA. SEA-independent energy flow formulations are indeed developed in [6-8].

A beautiful attempt to formulate a new thermodynamic theory bridging the gap between the classical thermodynamic and the theory of dynamical systems is contained in $[9,10]$. In this context, some relevant concepts, originally related to thermodynamics, arise naturally also in the analysis of dynamical systems: the energy equipartition and the existence of an entropy-like quantity. The first, indeed one of the cardinal elements of statistical thermodynamics, has been investigated in $[11,12,16]$ and has also points of contact with the topic of the present paper. The concept of entropy has been indeed proved to be consistent even in the frame of structural vibrations and acoustics [13-15]. Finally, partly related to the entropy concept, the question of irreversibility in structural dynamics, even in the absence of dissipation effects, has been considered in [16-19], with significant implications also on the practical design of special devices characterized by an energy trapping capability and energy sink storage. The problem of energy distribution along real structures has been also approached attacking the problem by dimensional analysis as shown in [20,21].

An additional area of investigation in this context is represented by the energy control through nonlinearities, the most active group in the field names this phenomenon energy pumping. A series of papers [22-25] are devoted to this topic and a general overview of the problem can be found in the book [26]. The authors clearly show how, especially non-smooth nonlinearities, can be used to design special nonlinear attachments to be coupled to a primary linear structure to damp its motion, subtracting a significant amount of vibration energy form the primary. This process again shows how the shock vibration energy can be confined into a subpart of the excited system.

An interesting and different point of view on the energy storage and energy re-distribution in complex systems, that belongs to a different context and research field, is indeed contained in [27-31]. Suitable structural modifications are there conceived coupling a mechanical structure to an electrical system. Under the dynamic point of view, the investigated electro-mechanical coupling is characterized by a single state vector collecting both mechanical and electrical degrees of freedom. The analysis developed by the authors, shows how it is possible to properly select some characteristics of the coupled dynamic matrix in a way most of the energy can be re-directed into the electrical degrees of freedom. In this case, an energy storage is physically confined into the electrical part of the system, but the essence of the process has the same aims of the present analysis: try to pilot the energy towards some selected degrees of freedom of a complex dynamic system. In this sense, the results here presented can have also potential applications in the context of design of an electrical shunt piezo-electrically coupled to a vibrating structure.

Finally, a special mention deserves the formation of regions of energy accumulation in continuous media, as a consequence of non-classical strain-stress relationships. In this context, deformation or kinetic energy can be concentrated in small regions through the addition of parts constituted by materials exhibiting a state of deformation effectively described by suitable tridimensional higher gradient continua as in [32,33] or two-dimensional structured continua as, e.g. in [34,35].

This paper proposes an additional contribution in this general context, providing a demonstration of a general energy decomposition property, that helps in simple understanding of localization and equaipartition of energy in complex structures, and that can be used as a design tool.

\section{An energy decomposition property}

\subsection{Undamped systems}

This section presents an energy decomposition property that holds for any linear elastic system, the basis for the shock analysis presented in Section 3. Initially an undamped structure is considered, and the analysis is later extended to dissipative systems.

Consider a conservative $N$-dof linear system and its usual equation of motion:

$$
\mathbf{M} \ddot{\mathbf{x}}+\mathbf{K x}=\mathbf{0}
$$


where $\mathbf{M}, \mathbf{K}$ and $\mathbf{x}$ are the mass, the stiffness matrix and the displacement vector, respectively. Introducing the transformation:

$$
\mathbf{x}(t)=\boldsymbol{\Phi q}(t)
$$

the eigenproblem equation is obtained:

\section{$\mathbf{K \Phi}=\mathbf{M \Phi \Lambda}$}

where $\mathbf{q}(t)$ and $\boldsymbol{\Phi}$ are the modal coordinates and the eigenvectors matrix, respectively, $\boldsymbol{\Lambda}$ is the eigenvalues matrix and $\omega_{j}=\sqrt{\boldsymbol{\Lambda}_{J J}}$ the $j$-th eigenfrequency. Under the assumption the mass matrix $\mathbf{M}$ is diagonal and has nonzero elements, let us introduce the vectors $\mathbf{U}_{\mathrm{j}}$ as:

$$
\mathbf{U}_{j}=\mathbf{M}^{1 / 2} \boldsymbol{\Phi}_{j}
$$

where $\boldsymbol{\Phi}_{j}$ is the $j$-th column of the eigenvector matrix, which leads to the symmetric eigenproblem:

$$
\mathbf{B U}=\mathbf{U} \boldsymbol{\Lambda}
$$

The matrix $\mathbf{B}=\mathbf{M}^{-1 / 2} \mathbf{K} \mathbf{M}^{-1 / 2}$ is symmetric and the orthonormality conditions hold:

$$
\begin{aligned}
& \mathbf{U}^{T} \mathbf{U}=\mathbf{I} \\
& \mathbf{U}^{T} \mathbf{B U}=\boldsymbol{\Lambda}
\end{aligned}
$$

For initial displacements equal to zero, the velocity vector is determined as:

$$
\dot{\mathbf{x}}=\sum_{j=1}^{N} \mathbf{U}_{j}^{T} \mathbf{M}^{-1 / 2} \dot{\eta}_{j}(t)
$$

where $\dot{\eta}_{j}(t)=\left(\mathbf{M V} \mathbf{M}^{-1 / 2} \mathbf{U}_{j}\right) \cos \left(\omega_{j} t\right)$ is the modal velocity and $\mathbf{V}_{0}$ the initial velocity vector.

Let introduce the covariance matrix:

$$
C_{\dot{\mathbf{x}}}=E\left\{\dot{\mathbf{x}}^{T}\right\}
$$

where $E\{\quad\}=\lim _{T \rightarrow \infty} \frac{1}{T} \int_{0}^{T} d t$ is the asymptotic mean time operator. Substitution of Eq. (4) into Eq. (5) leads to:

$$
C_{\dot{\mathbf{x}}}=\frac{\mathbf{M}^{-1 / 2}}{2} \sum_{j=1}^{N} \mathbf{U}_{j} \mathbf{U}_{j}^{T}\left(\left[\mathbf{U}^{-1}\right]_{j} \mathbf{M}^{1 / 2} V_{0}\right)^{2} \mathbf{M}^{-1 / 2}
$$

$\boldsymbol{\Lambda}_{v}$ is the diagonal matrix containing the initial modal velocities $\mathbf{v}_{i}=\left[\mathbf{U}^{-1}\right]_{i} \mathbf{M}^{1 / 2} \mathbf{V}_{0}$ and $\left[\mathbf{U}^{-1}\right]_{i}$ is the $i$-th row of the matrix and $\mathbf{U}^{-1}$

$$
\boldsymbol{\Lambda}_{v}=\left[\begin{array}{cccc}
v_{1}^{2} & 0 & \cdots & 0 \\
0 & v_{2}^{2} & & \vdots \\
\vdots & & & 0 \\
0 & \cdots & 0 & v_{\mathrm{N}}^{2}
\end{array}\right]
$$


that can be substituted into Eq. (6) to recast the covariance matrix in the form:

$$
C_{\dot{\mathbf{x}}}=\frac{\mathbf{M}^{-1 / 2}}{2} \mathbf{U} \boldsymbol{\Lambda}_{v} \mathbf{U}^{T} \mathbf{M}^{-1 / 2}
$$

Finally considering Eq. (1), the energy matrix $\mathbf{E}$ is introduced as:

$$
\mathbf{E}=\mathbf{M} C_{\dot{\mathbf{x}}}
$$

This matrix contains the information related to the average energy storage among the degrees of freedom, for initial velocities arbitrarily given for each degree of freedom or, equivalently, for an arbitrary set of impulses applied to the structure, with initial displacements equal zero.

With these preliminary positions we can illustrate a remarkable property of the energy matrix. Let us express the $r$-th element of the matrix $\boldsymbol{\Lambda}_{v}$ as a polynomial in terms of powers of the eigenvalues $\lambda_{r}$ :

$$
v_{r}^{2}=\sum_{i=0}^{N-1} \beta_{i} \lambda_{r}^{i}
$$

where $\beta_{i}$ are the coefficients of this polynomial expansion. Equation (9) in matrix form reads:

$$
\mathbf{w}=\boldsymbol{\Gamma} \boldsymbol{\beta}
$$

where $\mathbf{w}=\left(v_{1}^{2}, . ., v_{N}^{2}\right)^{T}, \boldsymbol{\Gamma}=\left[\begin{array}{cccc}1 & \lambda_{1} & \cdots & \lambda_{1}^{N-1} \\ 1 & \lambda_{2} & \cdots & \lambda_{2}^{N-1} \\ \vdots & \vdots & & \vdots \\ 1 & \lambda_{N} & \cdots & \lambda_{N}^{N-1}\end{array}\right]$ and $\boldsymbol{\beta}=\left(\beta_{1}, . ., \beta_{N}\right)^{T}$.

Since it is assumed the eigenvalues are distinct, $\boldsymbol{\Gamma}$ is a nonsingular Vandermonde matrix, and $\boldsymbol{\beta}$ is uniquely found by:

$$
\boldsymbol{\beta}=\boldsymbol{\Gamma}^{-1} \mathbf{w}
$$

meaning the decomposition Eq. (9) is unique. Therefore, $\boldsymbol{\Lambda}_{v}$ can be uniquely decomposed as a polynomial in terms of the eigenvalue matrix:

$$
\boldsymbol{\Lambda}_{v}=\sum_{i=0}^{N-1} \beta_{i} \boldsymbol{\Lambda}^{i}
$$

The orthonormality conditions in Eq. (3) lead to:

$$
\mathbf{B U}=\mathbf{U} \boldsymbol{\Lambda}
$$

then:

$$
\mathbf{\Lambda}=\mathbf{U}^{-1} \mathbf{B} \mathbf{U}
$$

Post-multiplication by $\boldsymbol{\Lambda}$ of Eq. (11) and use Eq. (12) produces:

$$
\mathbf{B U} \mathbf{\Lambda}=\mathbf{U} \mathbf{\Lambda}^{2} \rightarrow \mathbf{B}^{2} \mathbf{U}=\mathbf{U} \boldsymbol{\Lambda}^{2}
$$

Analogous iteration of the previous steps leads to: 


$$
\mathbf{\Lambda}^{q}=\mathbf{U}^{-1} \mathbf{B}^{q} \mathbf{U}
$$

Finally, substituting Eq. (7) into Eq. (8) and then using Eqs (10) and (13), after some algebra, the energy matrix is decomposed:

$$
\mathbf{E}^{T}=\mathbf{M}^{1 / 2}\left(\sum_{i=0}^{N-1} \frac{\beta_{i}}{2} \mathbf{B}^{i}\right) \mathbf{M}^{-1 / 2}
$$

Equation (14) represents the desired decomposition of the energy matrix. This equation allows relating the energy distribution along the structure directly to the mass and stiffness matrix of the system. Thus, in principle, this equation can be used to shape the matrix $\mathbf{B}$ to distribute the energy in the desired fashion. However, one can notice that the form of Eq. (14) is valid under special initial conditions, those expressed by Eq. (10). Although these conditions may represent, in general, complicated physical distribution of velocities, in some practical cases, as shown in the next sections, they can simplify into a localized velocity initial condition, well representing the effect of an impulse point force.

Application of this decomposition is illustrated in the next section.

\section{Energy localization and equipartitioning}

In the present section, the analysis of the energy distribution is carried on using Eq. (14). Of particular interest are the two extreme cases: energy localization, i.e. the presence of one energy focus, and the case of uniform distribution of energy, indeed the energy equipartitioning.

\subsection{Energy localization}

Equation (14) is employed to localize the initial energy into one, or more, degree of freedom, called energy focus.

Consider the special case for which the initial velocities matrix is equal to the eigenvalues matrix: $\boldsymbol{\Lambda}_{v} \equiv \boldsymbol{\Lambda}$, although particular, this case is important in the context of the present theory. In fact, in this case is simply $\boldsymbol{\beta}=(0,1,0 . ., 0)^{T}$, and introducing the dynamical matrix $\mathbf{A}=\mathbf{M}^{-1} \mathbf{K}$, Eq. (14) becomes:

$$
\mathbf{E}=\frac{\mathbf{A}}{2}
$$

Thus, if the following inequality is satisfied:

$$
A_{r r} \gg A_{i i} \quad \forall i \neq r
$$

Equation (15) leads to:

$$
E_{r r} \gg E_{i i} \quad \forall i \neq r
$$

that is the energy is localized into the $r$-th degrees of freedom, which is the energy focus of the system. The initial velocities are derived from the initial modal velocities:

$$
\mathbf{v}=\mathbf{U}^{-1} \mathbf{M}^{1 / 2} \mathbf{V}_{0} \rightarrow \mathbf{V}_{0}=\mathbf{M}^{-1 / 2} \mathbf{U v}
$$

and using Eq. (9): $v_{r}^{2}=\omega_{r}^{2}$, the initial velocity vector becomes:

$$
\mathbf{V}_{0}=\mathbf{M}^{-1 / 2} \sum_{i=1}^{N} \mathbf{U}_{i} \omega_{i}^{2}
$$


Set $m_{i}$ the $i$-th element along the diagonal of the mass matrix, the condition to satisfy Eq. (16) is simply:

$$
\frac{\mathbf{K}_{r r}}{m_{r}}>>\frac{\mathbf{K}_{i i}}{m_{i}} \forall i \neq r
$$

$\omega_{r}=\frac{\mathbf{K}_{r r}}{m_{r}}, \omega_{i}=\frac{\mathbf{K}_{i i}}{m_{i}}, \forall i \neq r$ can be interpreted as the natural frequency of oscillation of the $i$-th degrees of freedom when all the others are blocked. Therefore, Eq. (16) holds if initial conditions are those specified by Eq. (18) and if the natural frequency of the degree of freedom elected as the focus is much higher than any other frequency $\omega_{i}$.

The effectiveness of the localization process is indeed defined by the characteristic nondimensional frequency $\Omega=\omega_{r} / \omega_{i}$, which may be considered the quality factor of the process:

$$
\frac{E_{r r}}{E_{i i}}=\Omega^{2}
$$

Equation (15) discloses another remarkable property related to the energy storage in complex systems. Consider the whole system partitioned into two subsystems: the first of them, with $N_{1}$ dofs, has energy $E_{1}=\sum_{i=1}^{N_{1}} \frac{\mathbf{K}_{i i}}{m_{i}}$ and the second, with $N_{2}=N-N_{1}$ dofs, has energy $E_{2}=\sum_{i=N_{1}+1}^{N} \frac{\mathbf{K}_{i i}}{m_{i}}$. The fraction of energy stored in each subsystem is given by the ratio:

$$
\frac{E_{1}}{E_{2}}=\frac{\bar{\omega}_{1}^{2} N_{1}}{\bar{\omega}_{2}^{2} N_{2}}
$$

where:

$$
\bar{\omega}_{1}^{2}=\frac{1}{N_{1}} \sum_{i=1}^{N_{1}} \omega_{i}^{2}, \quad \bar{\omega}_{2}^{2}=\frac{1}{N_{2}} \sum_{i=N_{1}+1}^{N} \omega_{i}^{2}
$$

Equation (22) is interesting and suitable for comparison with some results found in Statistical Mechanics and Statistical Energy Analysis. In fact, it appears the energy is shared in proportion with the number of degrees of freedom of each subsystem, but proportionally also to the average of the square of the "blocked natural frequencies" of their individual masses.

\subsection{Equipartitioning}

The general analysis developed in Section 2 can be now applied to the opposite of the case investigated in Section 3.1: the case of energy equipartitioning.

The condition under which energy is equally shared among the resonators comes from Eq. (5), imposing:

$$
\mathbf{M} C_{\dot{\mathbf{x}}}=\frac{E_{0}}{N} \mathbf{I}
$$

Using Eq. (17), the total energy of the system $E_{0}$ can be written as function of the modal initial velocities as:

$$
E_{0}=\frac{\mathbf{M}}{2} \mathbf{V}_{0}^{T} \mathbf{I} \mathbf{V}_{0}=\frac{1}{2} \mathbf{v}^{T} \mathbf{U}^{T} \mathbf{M}^{-1} \mathbf{U} \mathbf{v}
$$

Combining Eqs (23) and (7), leads to: 


$$
\mathbf{U}^{-1} C_{\dot{\mathbf{x}}}=\frac{E_{0}}{N} \mathbf{U}^{T} \rightarrow \frac{1}{2}\left(\mathbf{U}^{T} \mathbf{U}\right) \boldsymbol{\Lambda}_{v} \mathbf{U}^{T}=\frac{E_{0}}{N} \mathbf{U}^{T}
$$

Finally, the use of ortogonality condition Eq. (3) produces:

$$
\boldsymbol{\Lambda}_{v}=\frac{2 E_{0}}{N} \mathbf{I}
$$

or:

$$
v_{i}=\sqrt{\frac{2 E_{0}}{N}}
$$

Equation (24) states the initial modal velocities have to be uniform to produce energy equipartition among the resonators.

\subsection{Numerical experiments and discussion}

The previous concepts are applied to a chain of resonators, to show how the energy decomposition property permits to build dynamical systems where the energy is asymptotically stored into the energy focus.

A chain of $N=20$ oscillators is considered; mass constants are uniform and equal to $m=1 \mathrm{~kg}$, while the stiffness distribution is:

$$
k_{i}=\left\{\begin{array}{l}
k=1 N / m \quad \forall i \neq r \\
k_{r} \quad i=r
\end{array}\right.
$$

as plotted in Fig. 1. Accordingly to Eqs (16) and (19), the $r-1$ and $r$-th degrees of freedom are the energy focuses of the system, because:

- the initial velocities matrix is set equal to the eigenvalues matrix: $\boldsymbol{\Lambda}_{v} \equiv \mathbf{\Lambda}$;

- the stiffness $k_{r}$ is set much larger than $k$, i.e. $k_{r}=100 k$.

In order to verify the accuracy of the previous equations, a numerical solution is computed, applying modal analysis and following the steps ahead:

a) eigenvectors and eigenvalues of the dynamical matrix $\mathbf{A}=\mathbf{M}^{-1} \mathbf{K}$ are evaluated;

b) using Eq. (20) the initial velocities $\mathbf{V}_{0}$ are evaluated;

c) using Eq. (4) the time velocity vector is computed, thus the mean time velocity $\overline{\mathbf{V}}$ is evaluated;

d) the time energy and the mean time energy stored in each resonator, $E=\mathbf{M V}^{T} \mathbf{I V} \quad \bar{E}=\mathbf{M} \overline{\mathbf{V}}^{T} \mathbf{I} \overline{\mathbf{V}}$, respectively, are then computed.

Setting $r=7$, Fig. 2 shows the main diagonal of $\mathbf{A}$ : it appears how the effect of the focus makes the square natural frequencies $\omega_{0 r-1}^{2}$ and $\omega_{0 r}^{2}$ much higher than the other ones, as explained in Section 3.1.

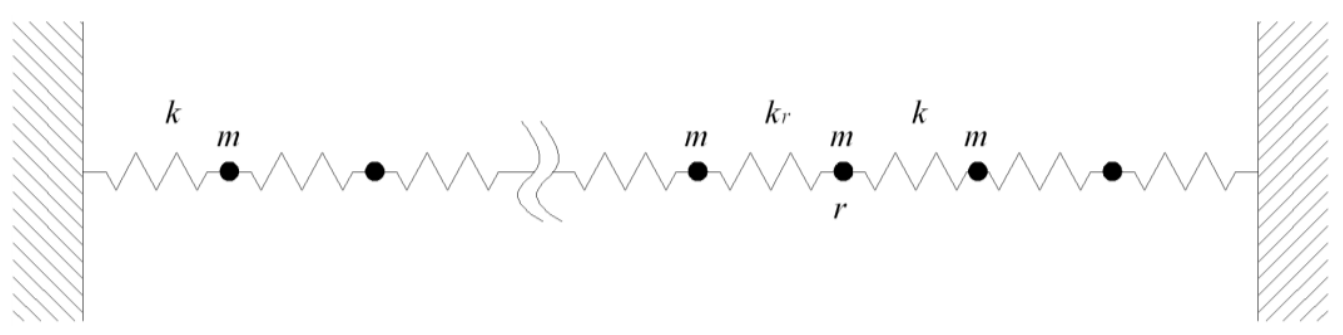

Fig. 1. Chain of resonators with stiffness focus. 


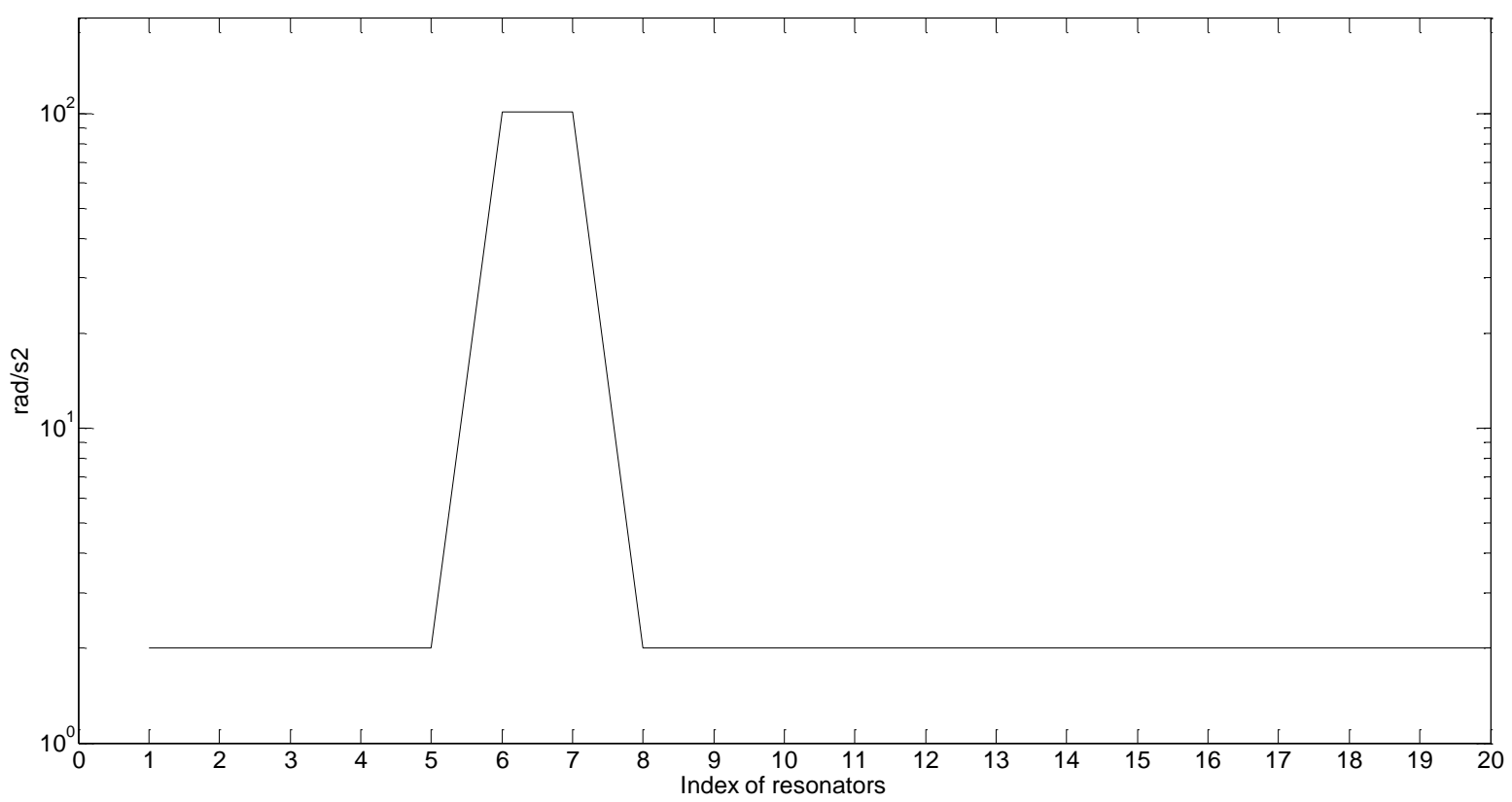

Fig. 2. Stiffness focus, $\operatorname{diag}(\mathbf{A})$ versus the mode index.

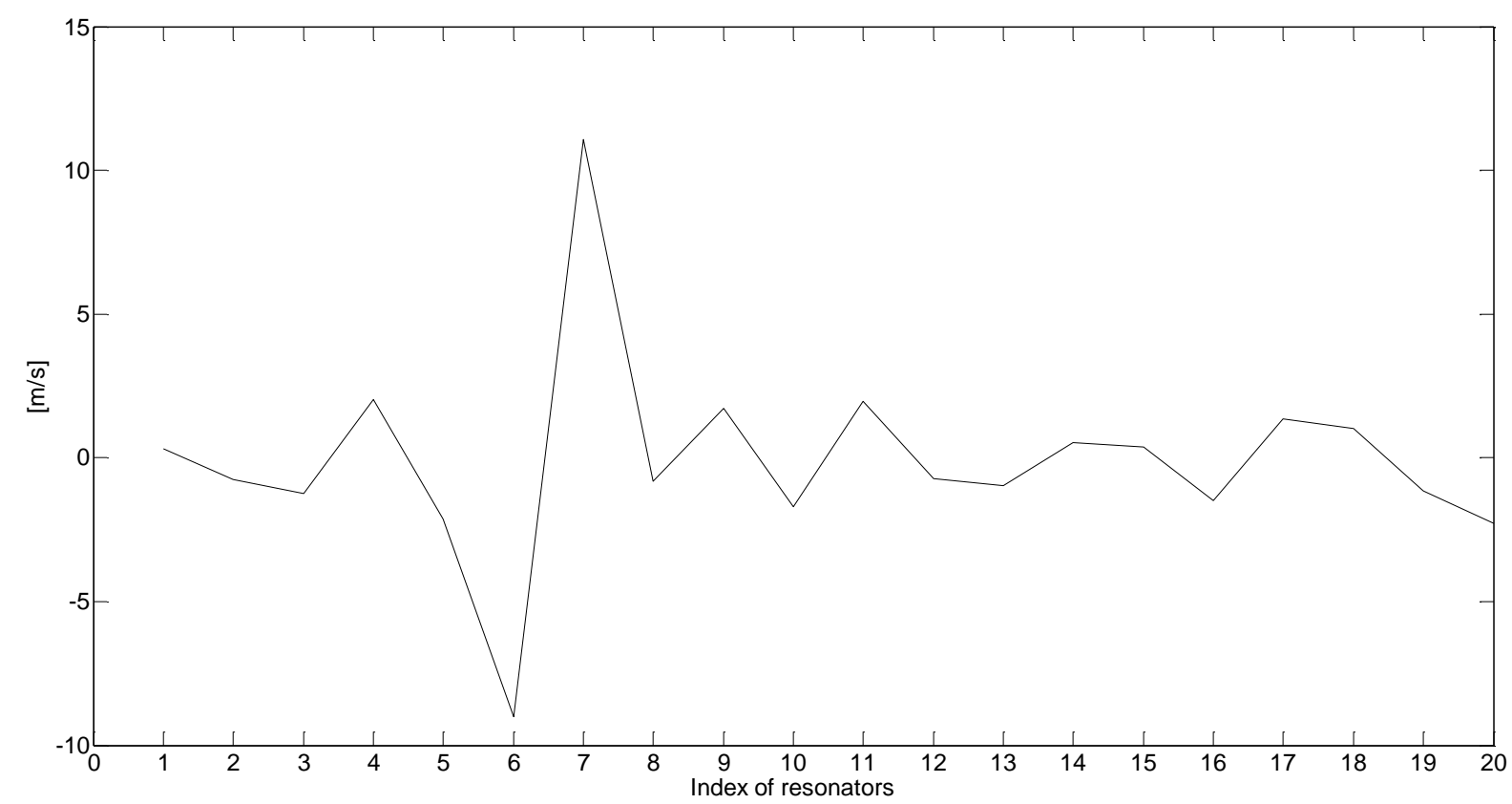

Fig. 3. Initial velocity vector versus mode index.

Figure 3 shows the initial velocity vector $\mathbf{V}_{0}$ : the modulus of the velocity imparted to $r-1$ and $r$-th degrees of freedom are higher than the ones imparted to the other resonators.

Figure 4 shows the moving average of the time energy $E(t)$, for comparison the moving average of a uniform chain of oscillators, i.e. mass and stiffness equal to $m$ and $k$, respectively, excited by the same initial conditions $\mathbf{V}_{0}$, is plotted in Fig. 5. The figures show: 


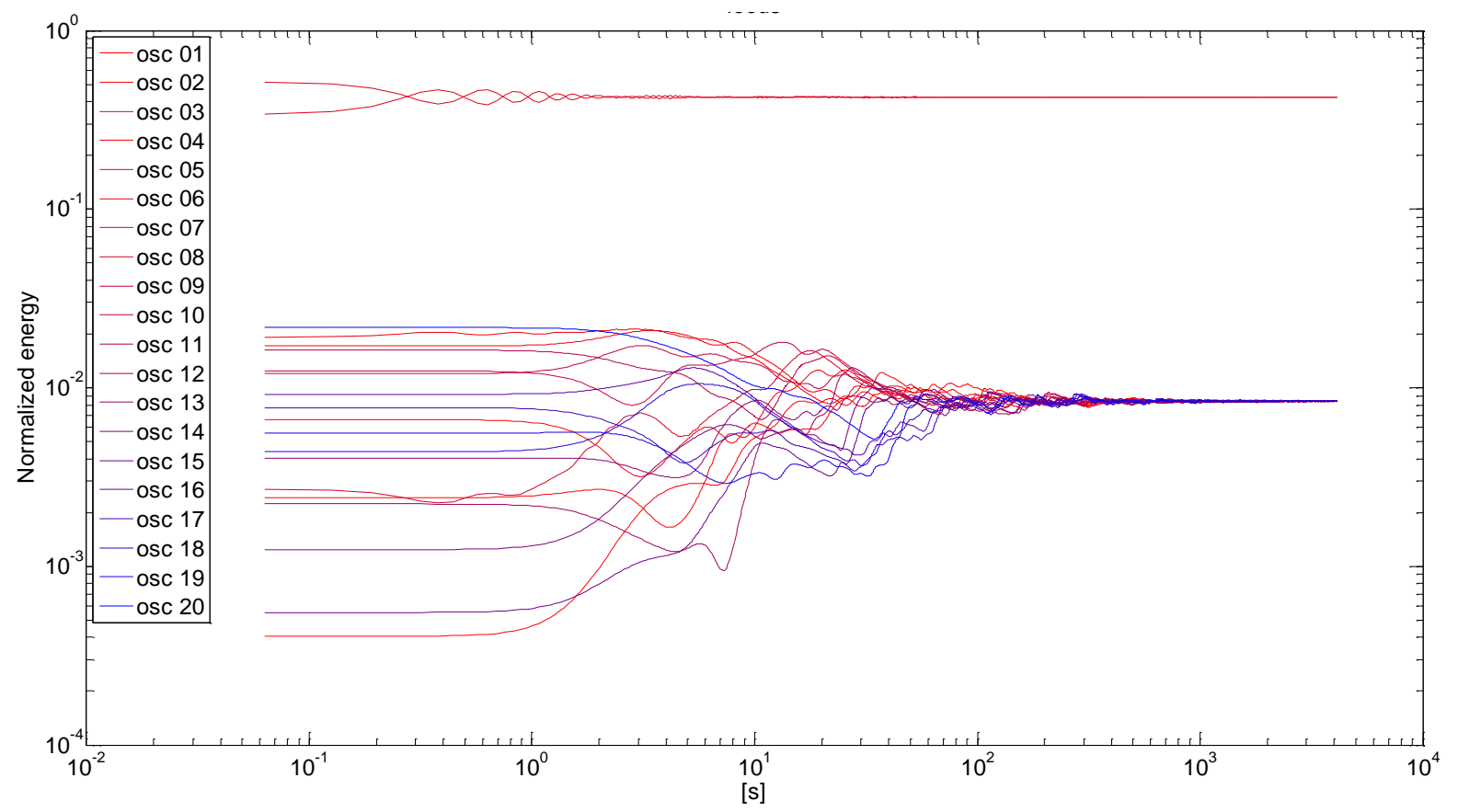

Fig. 4. Chain with stiffness focus, time energy averages versus time, $x, y$ axes in log scale.

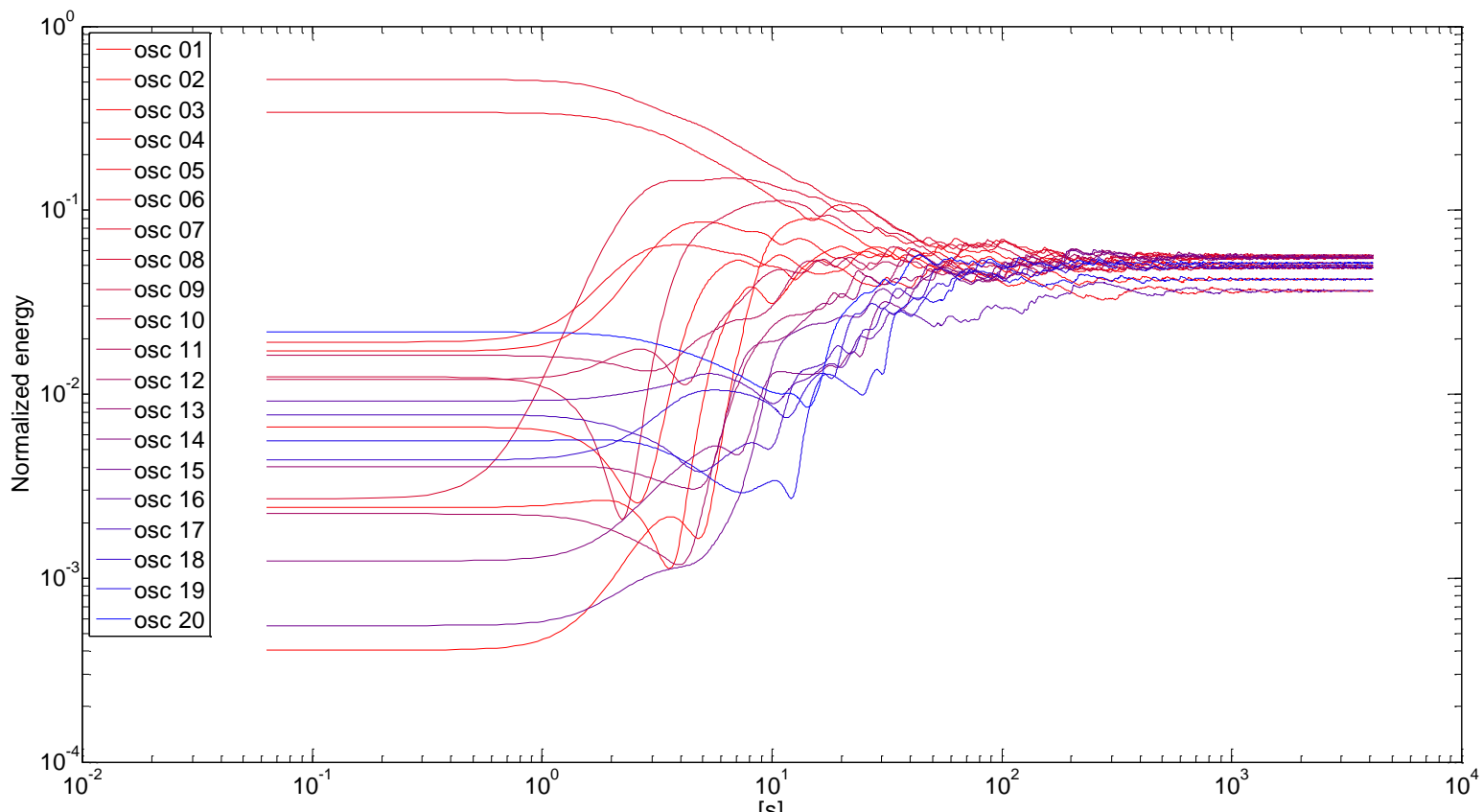

Fig. 5. Uniform chain, time energy averages versus time, $x, y$ axes in log scale.

- in the non-homogenous chain most of the energy remains stored into the focuses: the mean time energy of $r$ -1 and $r$-th degree of freedom is roughly fifty time greater than the other ones, as predicted by Eq. (15);

- in the homogenous chain, after an initial transient where the energy flows from the most excited resonators to the other ones, the energy is almost evenly shared among the resonators. 


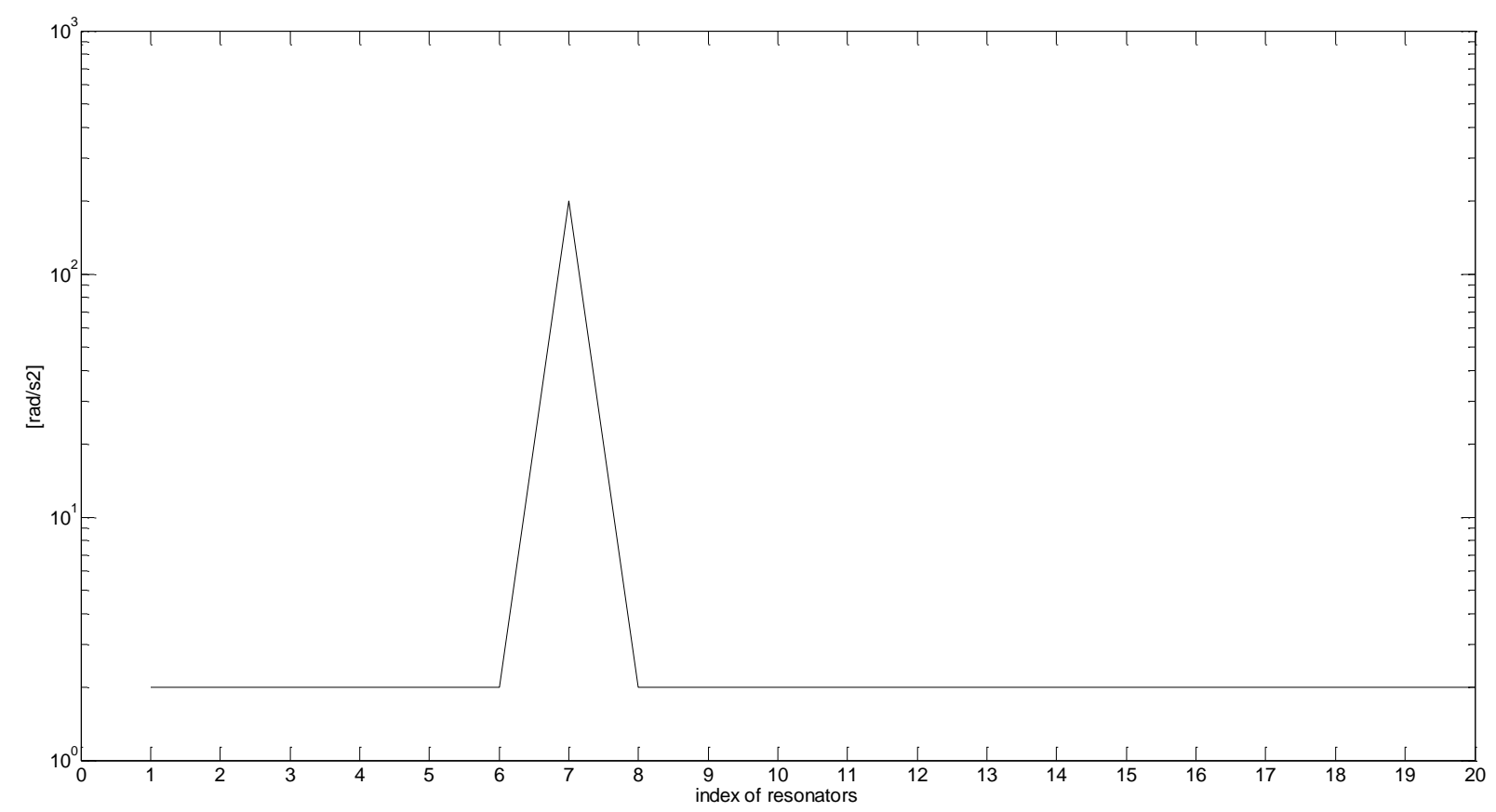

Fig. 6. Mass focus, $\operatorname{diag}(\mathbf{A})$ versus mode index.

The mass distribution:

$$
m_{i}=\left\{\begin{array}{l}
m \quad \forall i \neq r \\
m_{r}=\frac{m}{100} \quad i=r
\end{array}\right.
$$

can be considered for the following numerical analysis. The same steps listed in the previous e) $-\mathrm{h}$ ) points are performed.

Figure 6 shows the main diagonal of the dynamical matrix A: it appears how the small mass of the focus makes the square natural frequency $\omega_{0 r}^{2}$ much higher than other ones.

Figure 7 shows the initial velocity vector $\mathbf{V}_{0}$ : the velocity imparted to the focus is much higher than the ones imparted to the other resonators (roughly 100 times greater), thus the initial conditions are very close to an impulsive excitation of the focus only.

Figure 8 shows the moving average of the time energy $E(t)$, for comparison also a uniform chain of oscillators is considered, in Fig. 9. Even for the mass focus the figures enlighten:

- in the non-homogenous chain nearly the whole energy remains stored into the focus only: its mean time energy is roughly a hundred time greater than the other ones, as predicted by Eq. (15);

- in the homogenous chain, after an initial transient, the energy is almost evenly shared among resonators, at $t=0$ there are two symmetric peaks, the first is located at $r=7$, which is the most energetic degree of freedom at $t=0$, as Fig. 10 shows.

It is worthwhile to stress the total energy is higher for the uniform chain, as Fig. 10 shows, because (i) the total energy is roughly $E_{0} \approx \frac{\mathbf{V}_{0 r} m_{r}}{2}$, (ii) since $m_{r}=\frac{m}{100}$ in the non-homogeneous chain, it results:

$$
\frac{E_{0}^{h m g}}{E_{0}^{f o c}} \approx \frac{m}{m_{r}}=\frac{1}{100}
$$




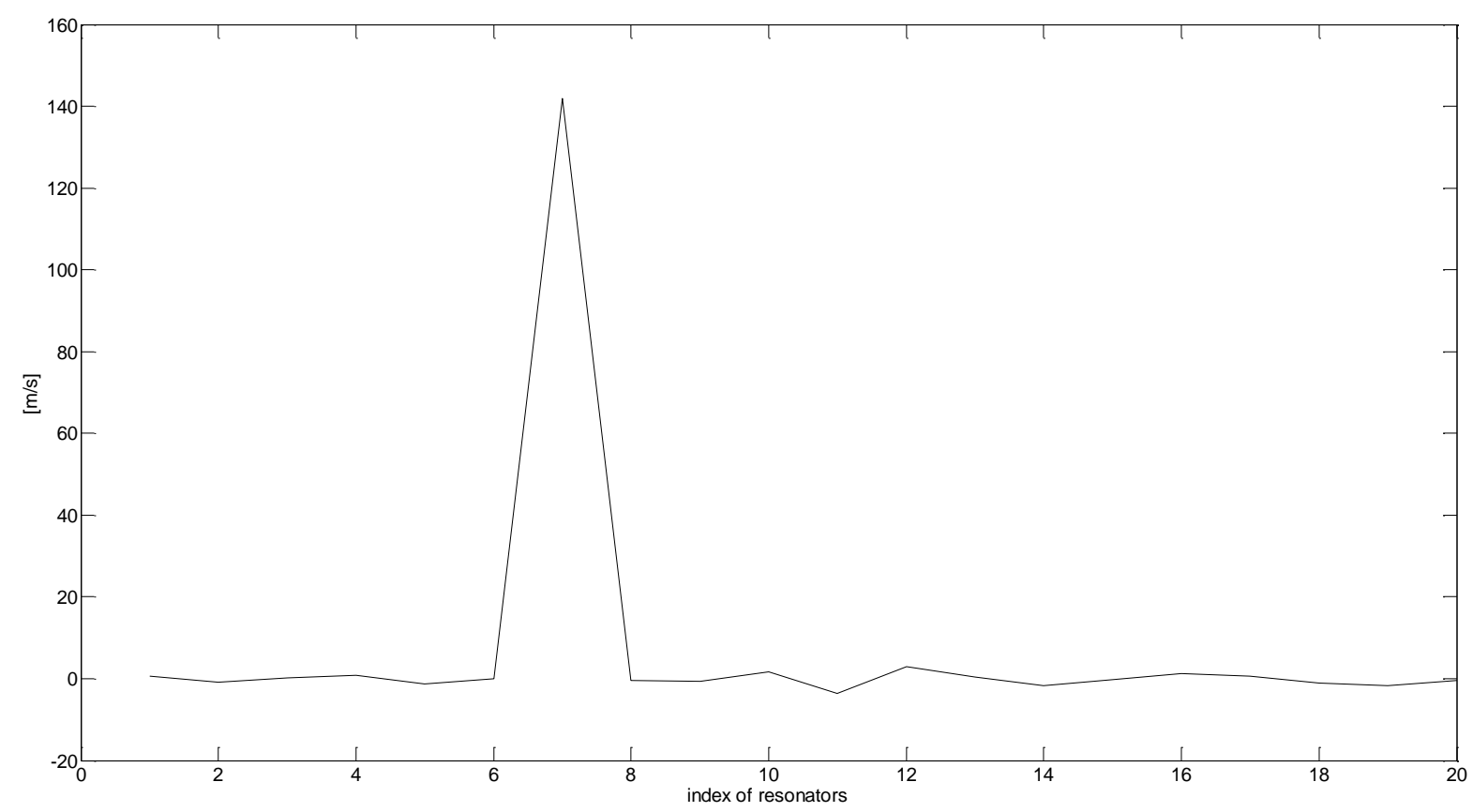

Fig. 7. Initial velocity vector versus mode index.

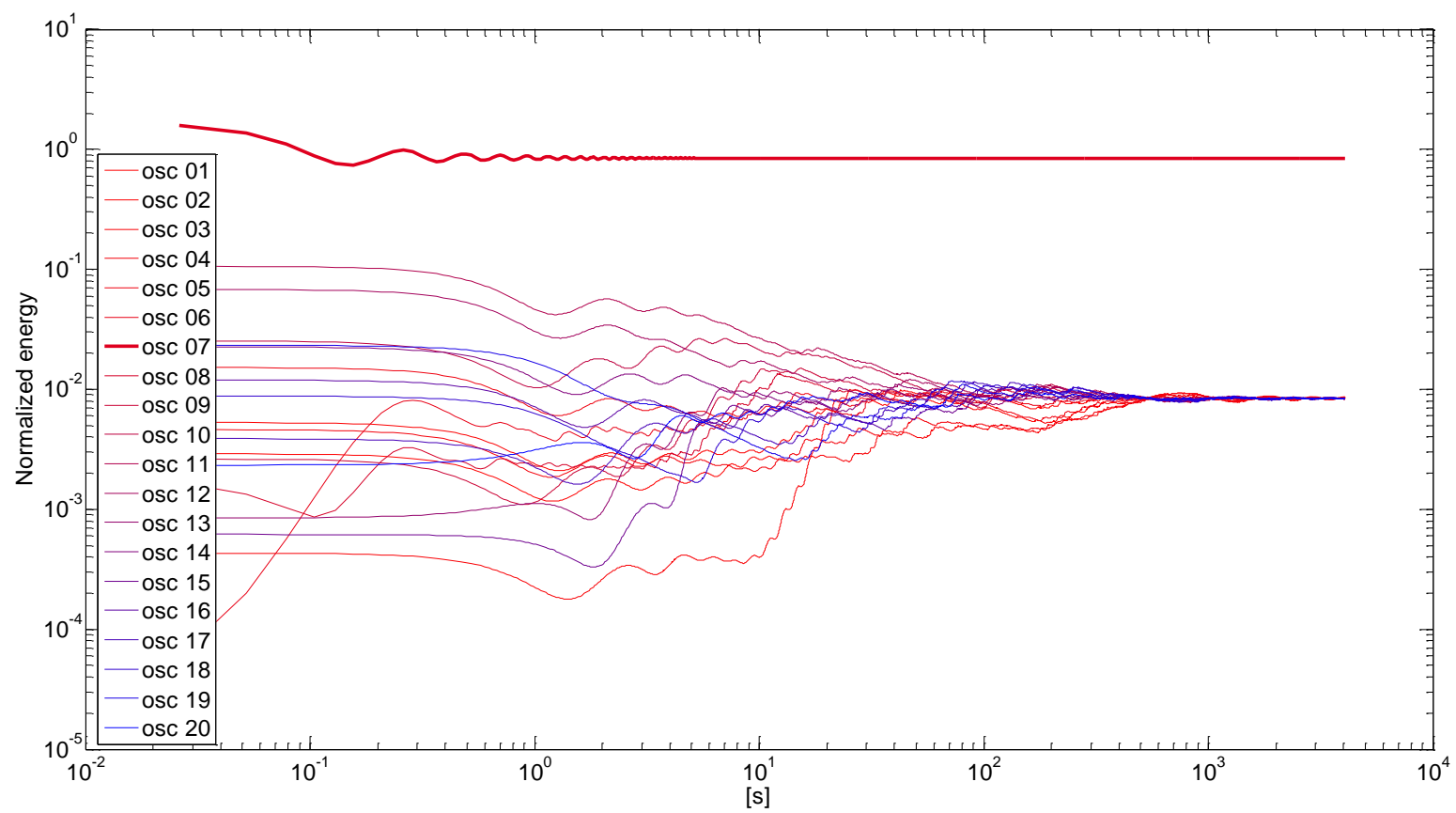

Fig. 8. Chain with mass focus, time energy averages versus time, $x, y$ axes in log scale.

Finally, the accuracy of Eq. (21) is verified. In case of uniform spring constants Eq. (21) becomes:

$$
\frac{E_{1}}{E_{2}}=\frac{\bar{m}_{2} N_{1}}{\bar{m}_{1} N_{2}}
$$




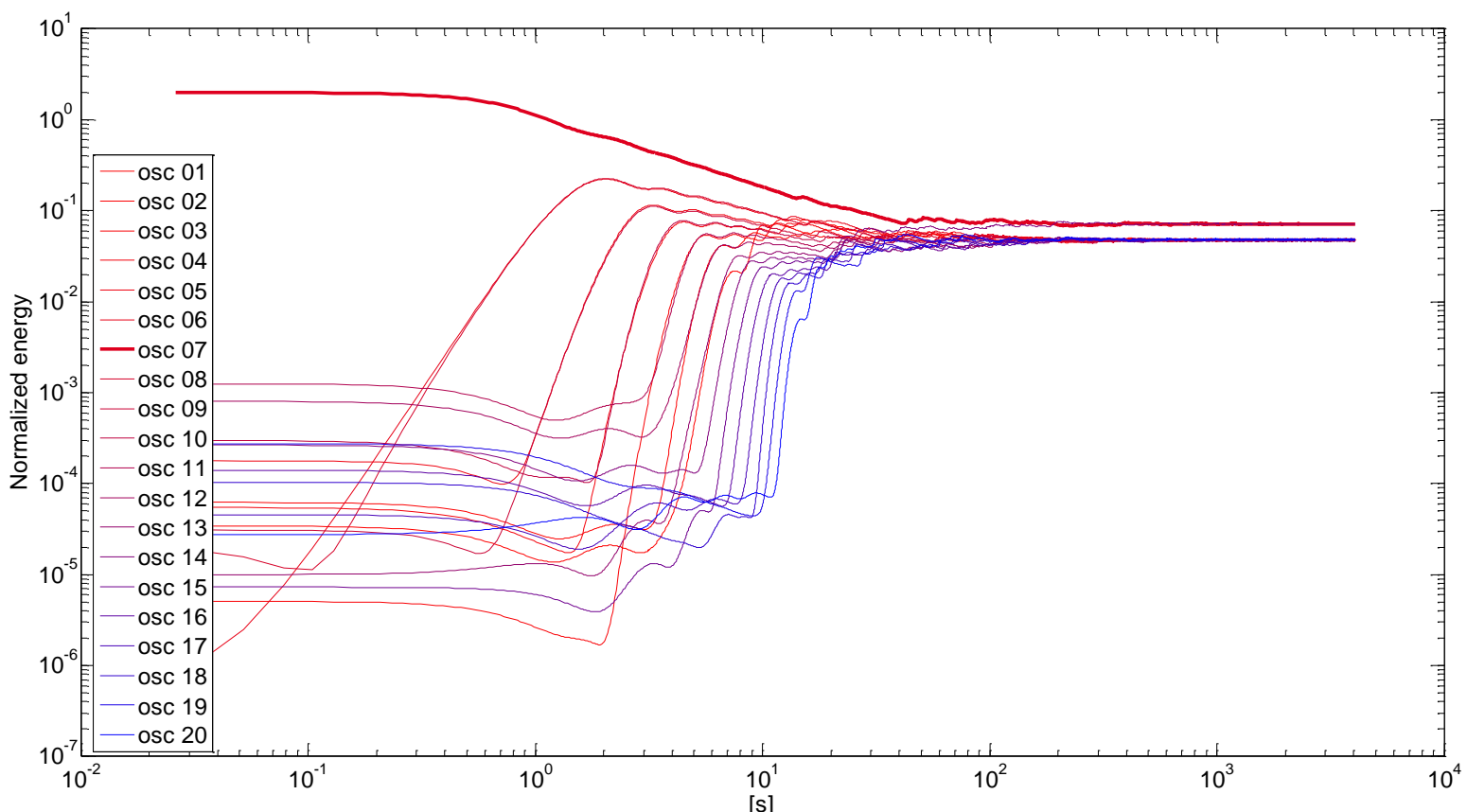

Fig. 9. Uniform chain, time energy averages versus time, $x, y$ axes in log scale.

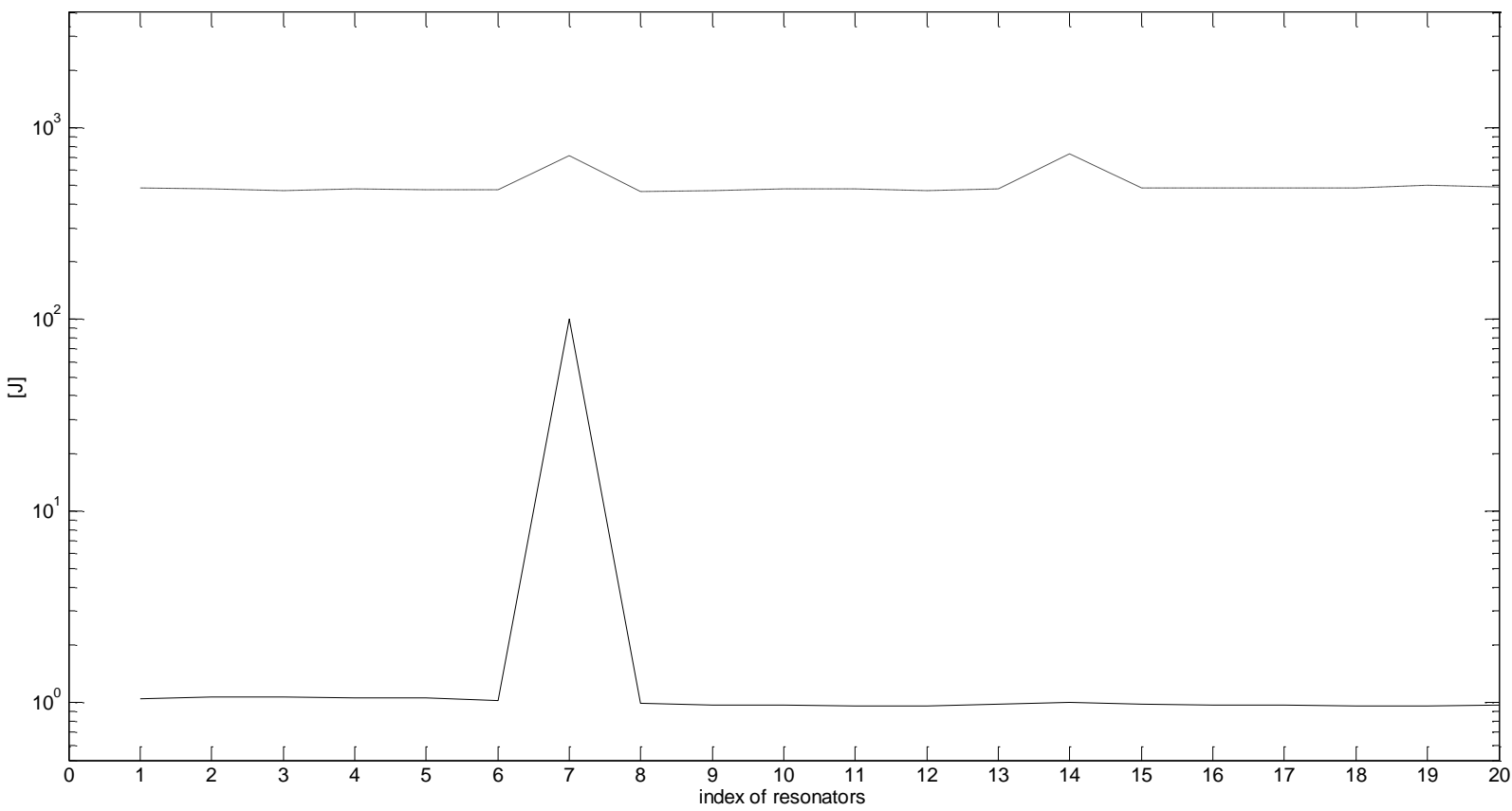

Fig. 10. Initial energy stored in the chain with the mass focus and in the uniform chain, in solid and dashed lines, respectively.

where $\bar{m}_{1}=\left(\frac{1}{N_{1}} \sum_{i=1}^{N} \frac{1}{m_{i}}\right)^{-1}$ and $\bar{m}_{2}=\left(\frac{1}{N_{2}} \sum_{i=N_{1}+1}^{N} \frac{1}{m_{i}}\right)^{-1}$ are the mean square mass in each subsystem. The mean time energies $\bar{E}_{1}$ and $\bar{E}_{2}$ are evaluated from the knowledge of $\bar{E}$ : 


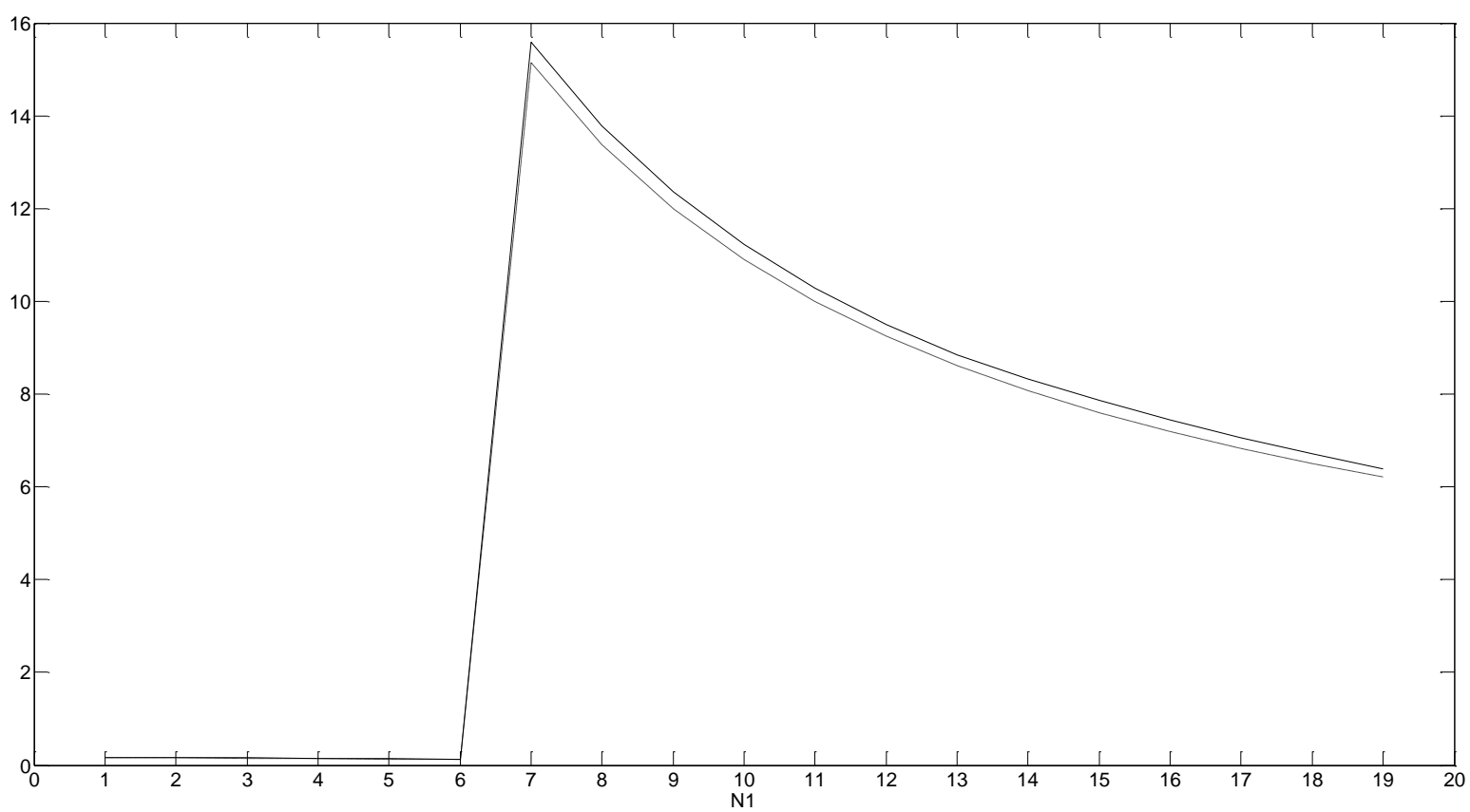

Fig. 11. Theoretical and numerical subsystems energy ratio, $\bar{E}_{1} / \bar{E}_{2}$, versus $N_{1}$, in solid and dashed lines, respectively.

$$
\bar{E}_{1}=\sum_{i=1}^{N_{1}} \overline{\mathbf{E}}_{i} \quad \bar{E}_{2}=\sum_{i=N_{1}+1}^{N} \overline{\mathbf{E}}_{i}
$$

Figure 11 shows a comparison between the ratio $\bar{E}_{1} / \bar{E}_{2}$ and the right-hand side of Eq. (25) plotted versus $N_{1}$ : the energy repartition among the subsystems predicted by Eq. (21) is in good agreement with numerical results; the energy is mainly localized in the subsystem which contains the focus.

Even considering Eqs (3) and (14) do not strictly hold in case of a mass focus, the previous results have shown Eqs (15) and (19) are in very good agreement with numerical experiments.

\section{Damped systems}

The previous results, although originally obtained for undamped systems, are extended to dissipative systems, using the same assumptions under which Eq. (15) holds. Equation (1) becomes:

$$
\mathbf{M} \ddot{\mathbf{x}}+\mathbf{C} \dot{\mathbf{x}}+\mathbf{K x}=\mathbf{0}
$$

where $\mathbf{C}$ is the damping matrix.

Consider firstly proportional damping:

$$
\mathbf{C}=a \mathbf{M}+b \mathbf{K}
$$

where $a$ and $b$ are real constants. Thus:

$$
\mathbf{U}^{T} \mathbf{C U}=a \mathbf{I}+b \boldsymbol{\Lambda}
$$

where the modal damping coefficients $\mathbf{C}_{j j}$ are: 


$$
\mathbf{C}_{j j}=a+b \omega_{j}^{2}=2 \gamma_{j} \omega_{j}
$$

Using modal analysis the displacement vector is $\mathbf{x}=\mathbf{U} \eta(t)$, and the modal coordinates are:

$$
\eta_{d i}(t)=\frac{\mathbf{v}_{i}}{\omega_{d i}} e^{-\gamma_{i} \omega_{i} t} \sin \left(\omega_{d i} t\right)
$$

where $\omega_{d i}=\omega_{i} \sqrt{1-\gamma_{i}^{2}}$ and $\mathbf{v}_{i}=\left[\mathbf{U}^{-1}\right]_{i} \mathbf{V}_{0}$, the modal velocities are:

$$
\dot{\eta}_{d i}(t) \approx e^{-\gamma \omega_{i} t} \mathbf{v}_{i} \cos \left(\omega_{d i} t\right) \approx e^{-\gamma \omega_{i} t} \dot{\eta}_{u i}(t)
$$

where $\dot{\eta}_{u i}(t)$ is the modal velocity for $\mathbf{C}=\mathbf{0}$.

Equation (26) enlightens the obvious fact proportional damping causes an exponential attenuation of the vibration amplitude, governed by the time constants $\tau_{i}=1 /\left(\gamma \omega_{i}\right)$. It follows the covariance matrix defined by Eq. (5) is zero: a different definition is indeed required. Let $E_{T}\{\}=\frac{1}{T} \int_{0}^{T} d t$ the mean time operator over a finite time $T$, the covariance matrix becomes:

$$
C_{\dot{\mathbf{x}}}=\sum_{i=1}^{N} \sum_{j=1}^{N} \mathbf{U}_{j} \mathbf{U}_{i}^{T} \mathbf{v}_{i} \mathbf{v}_{j} E_{T}\left\{e^{-\gamma \omega_{i} t} e^{-\gamma \omega_{j} t} \cos \left(\omega_{d i} t\right) \cos \left(\omega_{d j} t\right)\right\}
$$

considering $\gamma \ll 1$ :

$$
E_{T}\left\{e^{-\gamma \omega_{i} t} e^{-\gamma \omega_{j} t} \cos \left(\omega_{d i} t\right) \cos \left(\omega_{d j} t\right)\right\} \approx \frac{1}{4 T \gamma \omega_{i}} \delta_{i j}=\frac{\tau_{i}}{4 T} \delta_{i j}
$$

where $\delta_{i j}$ is the Kronecker delta.

With the position:

$$
D=\frac{1}{2}\left[\begin{array}{cccc}
\tau_{1} & 0 & \cdots & 0 \\
0 & \tau_{2} & & \vdots \\
\vdots & & & 0 \\
0 & \cdots & 0 & \tau_{N}
\end{array}\right]
$$

the covariance matrix takes the form:

$$
C_{T \dot{\mathbf{x}}}=\frac{1}{2 T} \mathbf{U D} \boldsymbol{\Lambda}_{v} \mathbf{U}^{T}
$$

Considering $\boldsymbol{\Lambda}_{v} \equiv \boldsymbol{\Lambda}$, after some algebra the energy matrix of the damped system becomes:

$$
\mathbf{E}_{d}=\frac{1}{T} \mathbf{E}_{u} \Pi
$$

where $\Pi=\mathbf{U D \mathbf { U } ^ { T }}$ and $\mathbf{E}_{u}^{T}$ is the energy matrix of the undamped system, given by Eq. (15).

To obtain insights into the effect of damping in the energy distribution, the structure of the matrix $\Pi$ has to be investigated. Considering the eigenfrequencies in ascending order, the following approximations are introduced: 


$$
\begin{gathered}
\tau_{N}=\frac{1}{\gamma \omega_{N}} \approx \frac{1}{\gamma} \sqrt{\frac{m_{r}}{k_{r}}} \\
\tau_{i} \approx \frac{1}{\gamma} \sqrt{\frac{m}{k}}, \forall i \neq N
\end{gathered}
$$

the matrix $\mathbf{D}$ is rearranged accordingly:

$$
D \approx \frac{1}{2 \gamma} \sqrt{\frac{m}{k}}\left(\left[\begin{array}{cccc}
1 & 0 & \cdots & 0 \\
0 & 1 & & \vdots \\
\vdots & & & 0 \\
0 & \cdots & 0 & 1
\end{array}\right]-\left(1-\frac{1}{\Omega}\right)\left[\begin{array}{cccc}
0 & 0 & \cdots & 0 \\
0 & 0 & & \vdots \\
\vdots & & & 0 \\
0 & \cdots & 0 & 1
\end{array}\right]\right)=c_{1} \mathbf{I}-c_{2} \mathbf{O}_{N}
$$

where $c_{1}=\frac{1}{2 \gamma} \sqrt{\frac{m}{k}}, c_{2} \frac{1}{2 \gamma}\left(\sqrt{\frac{m}{k}}-\sqrt{\frac{m_{r}}{k_{r}}}\right), \mathbf{I}$ is the identity matrix and:

$$
\mathbf{O}_{s}=\left\{\begin{array}{l}
\left(\mathbf{O}_{s}\right)_{i j}=0, \forall i \neq j \\
\left(\mathbf{O}_{s}\right)_{i j}=0, \forall i=j \neq s \\
\left(\mathbf{O}_{s}\right)_{i j}=1, \forall i=j=s
\end{array}\right.
$$

Substituting Eq. (30) into $\Pi=\mathbf{U D U}^{T}$, and considering that $\mathbf{U}_{r N} \gg \mathbf{U}_{i j} \forall i, j$, produces:

$$
\Pi \approx\left(c_{1} \mathbf{I}-u c_{2} \mathbf{O}_{r}\right)
$$

where $u=\mathbf{U}_{r N}^{2}$, and the orthonormality condition $u \approx 1$ is used. Finally, substituting Eq. (31) into Eq. (29) and considering Eq. (15) produces:

$$
\mathbf{E}_{d} \approx \frac{1}{T} \mathbf{E}_{u}\left(c_{1} \mathbf{I}-c_{2} \mathbf{O}_{r}\right)
$$

Equation (32) provides the final picture about the damping effect on energy partitioning: the first contribution reproduces the energy matrix in the absence of dissipation, while the second reduces the energy peak reached at the focus location. On this basis, it is relevant to introduce a comparison between the energy levels at the focus in the undamped and damped case, respectively, considering the energy storage ratio:

$$
\frac{\mathbf{e}_{d r}}{\mathbf{e}_{d i}} \approx \frac{1}{\Omega} \frac{\mathbf{e}_{u r}}{\mathbf{e}_{u i}} \approx \Omega
$$

where $\mathbf{e}_{d r}$ and $\mathbf{e}_{d i}$ are the mean time energy stored into the focus and into any other resonator of the damped system, respectively, $\mathbf{e}_{u r}$ and $\mathbf{e}_{u i}$ the corresponding quantities for the undamped case, and $\Omega \gg 1$ is the quality factor of the localization process.

The previous results show:

- the presence of damping produces energy coupling among the physical resonators, which reduces the capability of localizing energy into the focus;

- the energy localization reduction depends on $\Omega$;

- comparison of Eq. (33) with Eq. (20) shows the energy storage ratio is an order of magnitude smaller in presence of small damping. 
To complete the discussion, some other special cases of damping are considered. Uniform damping coefficient $c$ all along the chain produce:

$$
\mathbf{C}=c\left[\begin{array}{cccccc}
2 & -1 & 0 & \cdots & & 0 \\
-1 & 2 & -1 & & & \\
0 & \ddots & \ddots & \ddots & & \vdots \\
& & & & & 0 \\
\vdots & & & -1 & 2 & -1 \\
0 & & \cdots & 0 & -1 & 2
\end{array}\right]
$$

The ratio between the nondimensional damping coefficient of the focus, i.e. $\gamma_{r} \approx c / 2 m_{r} \sqrt{\frac{k_{r}}{m_{r}}}$, and the one associated to any other resonator, i.e. $\gamma \approx c / 2 m \sqrt{\frac{k}{m}}$, is:

$$
\frac{\gamma_{r}}{\gamma} \approx \sqrt{\frac{m k}{m_{r} k_{r}}}
$$

\section{Equation (34) enlightens:}

- when a mass focus is considered, the motion of the focus is over-damped, since $\gamma_{r} \gg \gamma$;

- when a stiffness focus is employed, the motion is under-damped, since $\gamma_{r} \ll \gamma$.

In this case it is concluded that the energy initially injected into the focus is quickly dissipated in case of a mass focus, while remains confined into it, nearly completely and for a long time accordingly to Eqs (16) and (20), in case of a stiffness focus.

Another case of damping is obtained for uniform nondimensional damping coefficient $\gamma$ along the chain:

$$
\mathbf{C}=\left[\begin{array}{cccccc}
2 c & -c & 0 & \ldots & & 0 \\
\ddots & \ddots & \ddots & & & \\
& -c & 2 c & -c_{r} & & \vdots \\
& & -c_{r} & 2 c_{r} & -c_{r} & \\
\vdots & & & -c_{r} & 2 c & -c \\
0 & & \ldots & & &
\end{array}\right]
$$

where $c_{r}$ is the damping coefficient of the focus. Since $\gamma$ is uniform, the validity of Eq. (33) can be extended to this case also, where the ratio $c_{r} / c$ becomes:

$$
\frac{c_{r}}{c}=\sqrt{\frac{m_{r} k_{r}}{m k}}
$$

\subsection{Numerical studies}

Theoretical results obtained for damped systems are corroborated with numerical experiments in this section, studying the chain oscillators equipped with a mass focus, with $m_{r}=m / 100$, and $r=7$. 


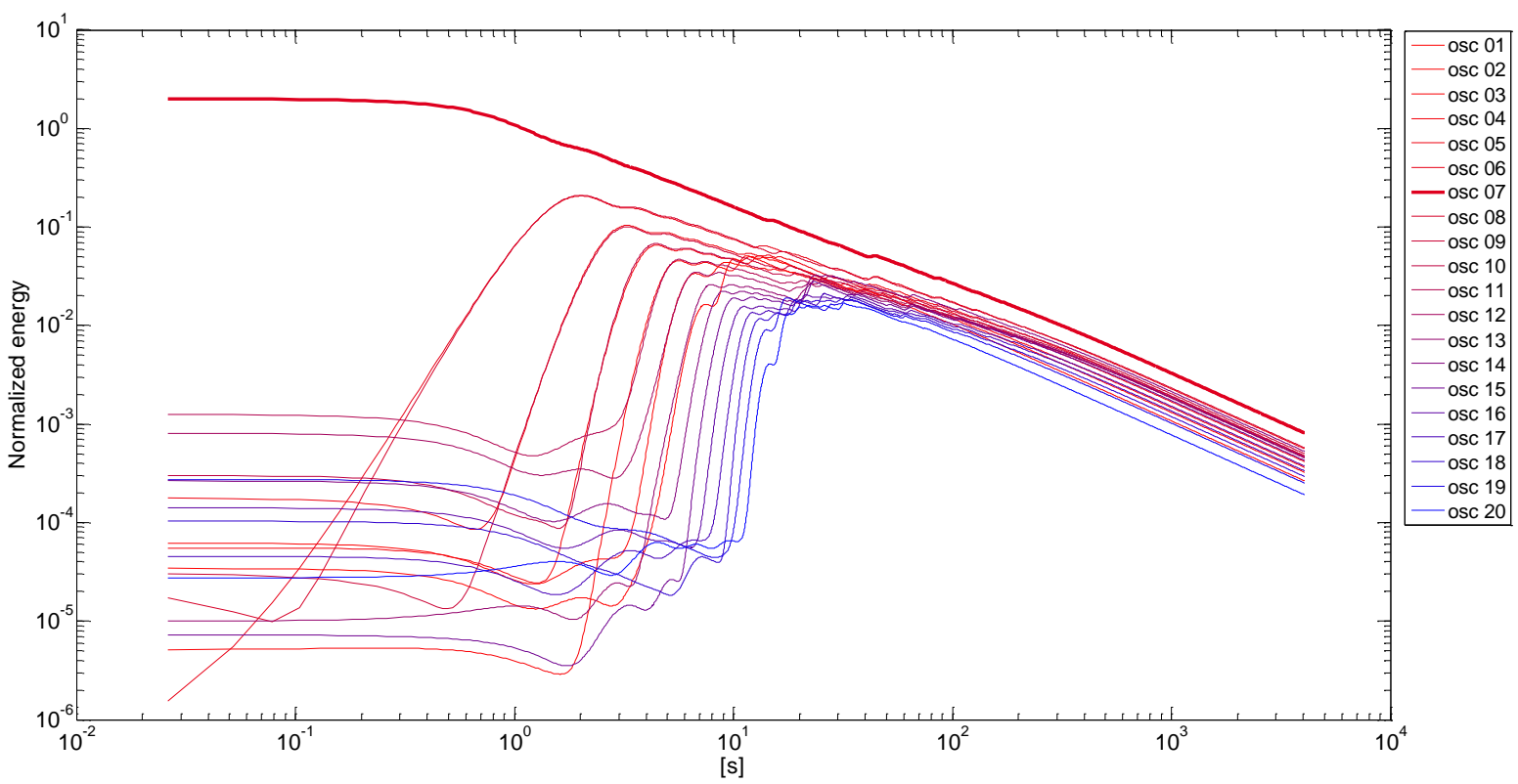

Fig. 12. Uniform chain with proportional damping, time energy averages versus time, $x, y$ axes in log scale.

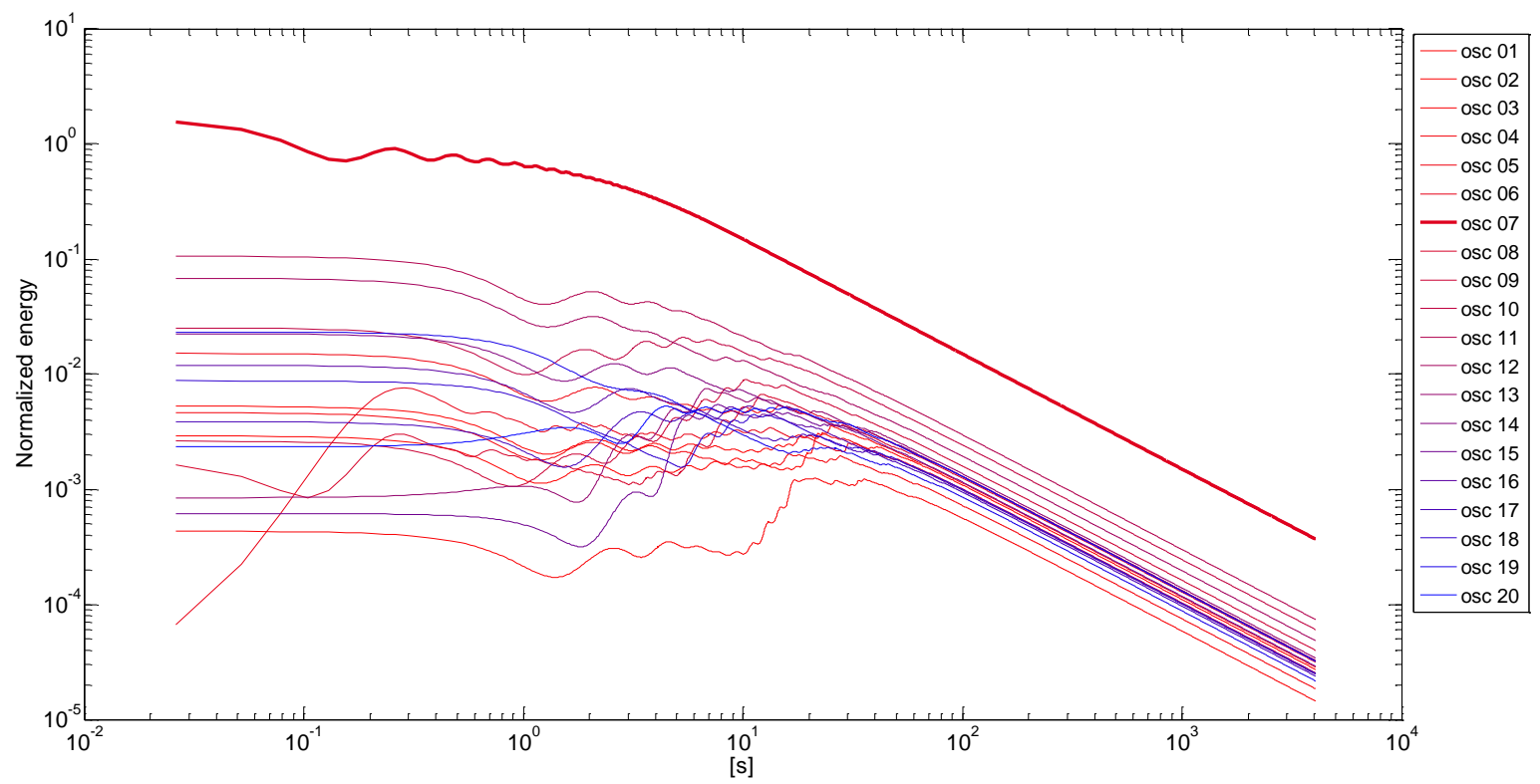

Fig. 13. Chain with mass focus with proportional damping, time energy averages versus time, $x, y$ axes in log scale.

Light proportional damping is firstly considered, setting $\gamma=0.02$. Figures 12 and 13 show the moving averages of the uniform and non-uniform chain of oscillators, respectively:

- the uniform chain is characterized by an initial diffusive process, whose time constant is roughly $\tau_{u}=\frac{1}{\gamma} \sqrt{\frac{m}{k}}=50 s$. After that, the moving average of any resonator linearly decreases with slope equal to $t$ in the bi-logarithmic diagram, as predicted by Eqs (26) and (27);

- the non-uniform chain is characterized by a two-phase diffusive process, whose time constants are 
$\tau_{f} \frac{1}{\gamma} \sqrt{\frac{m_{r}}{k}}=5 s$ and $\tau_{u}$. Up to the time $\tau_{f}$ the energy flows from the focus to the resonators then, while the moving average of the focus decreases as predicted by Eq. (27), the diffusive process interests only the other resonators, up to the time $\tau_{u}$.

- From the comparison of Figs 8 and 13, the energy storage ratios are roughly $10^{2}$ and $10^{1}$ for the conservative and the damped system, respectively. Since $\Omega=\sqrt{\frac{m}{m_{r}}}=\frac{1}{10}$, the prediction made by Eq. (33) agrees, quite accurately, with numerical data, i.e. $\frac{\mathbf{e}_{d r}}{\mathbf{e}_{d i}} / \frac{\mathbf{e}_{u r}}{\mathbf{e}_{u i}} \approx \frac{1}{\Omega}=\frac{1}{10} \equiv \frac{10^{1}}{10^{2}}$;

A suitable index which enlightens the energy coupling induced by the damping is defined ahead:

$$
\vartheta(t)=\sqrt{\frac{1}{N} \sum_{i=1}^{N}\left(\frac{e_{i}(t)-\bar{e}(t)}{\bar{e}(t)}\right)^{2}}
$$

where $e_{i}(t)$ is the moving average of the time energy of the $i$-th resonator, evaluated at the time $t$, while $\bar{e}(t)=\sum_{i=1}^{N} \frac{e_{i}(t)}{N}$ is the ensemble average. Equation (35) provides information about the spreading of energy around $\bar{e}(t)$, being $\vartheta(t)$ the RMS of the resonators' energies: if $\vartheta(t)=0$, the energy is equally shared among the resonators, when $\vartheta(t)=1$ the energy spreading is of the same order of the ensemble average $\bar{e}(t)$. Since the RMS is evaluated over moving averages, $\vartheta(t)$ provides information on the energy relaxation attitude that is a measure of the tendency of the system to energy equipartitioning.

$\vartheta(t)$ is plotted, on top and bottom subplots, in Fig. 14 for undamped and damped systems, respectively:

- damping does not substantially modify the energy sharing process in the uniform chain;

- considering the dissipative chain with the focus, part of the energy is diffused from the focus to the other resonators. Damping induces indeed an energy coupling among the resonators, which does not indeed take place in the conservative case;

- the presence of a double time-scale process, characterized by time constants, $\tau_{f}$ and $\tau_{u}$, clearly appears.
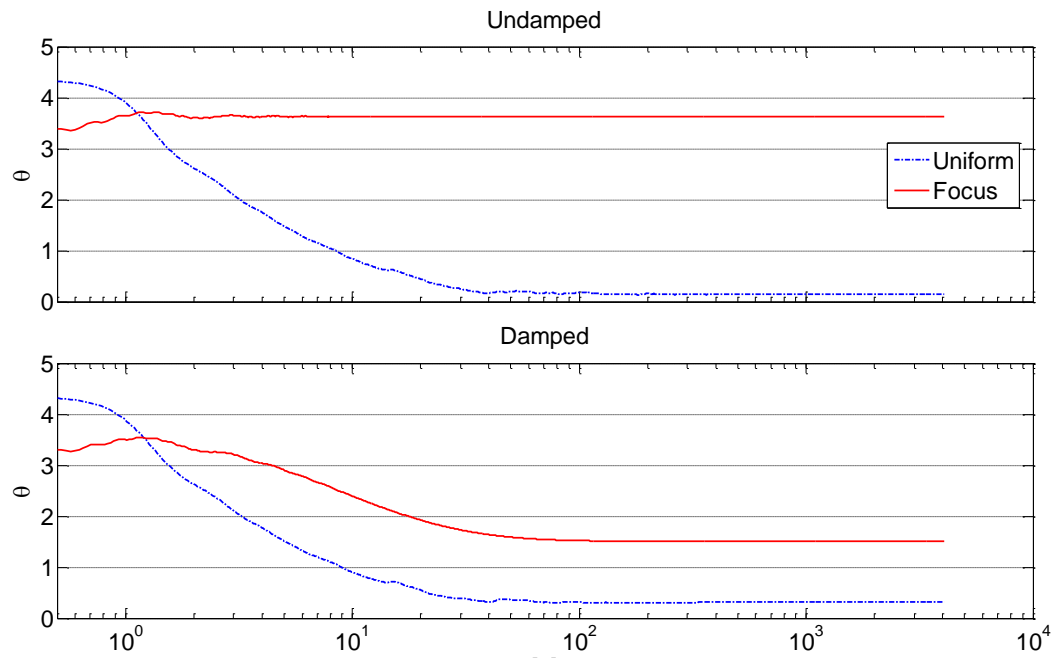

[s]

Fig. 14. Energy coupling index versus time, $x, y$ axes in log scale, for undamped and damped systems, in subplot on top and bottom, respectively. 


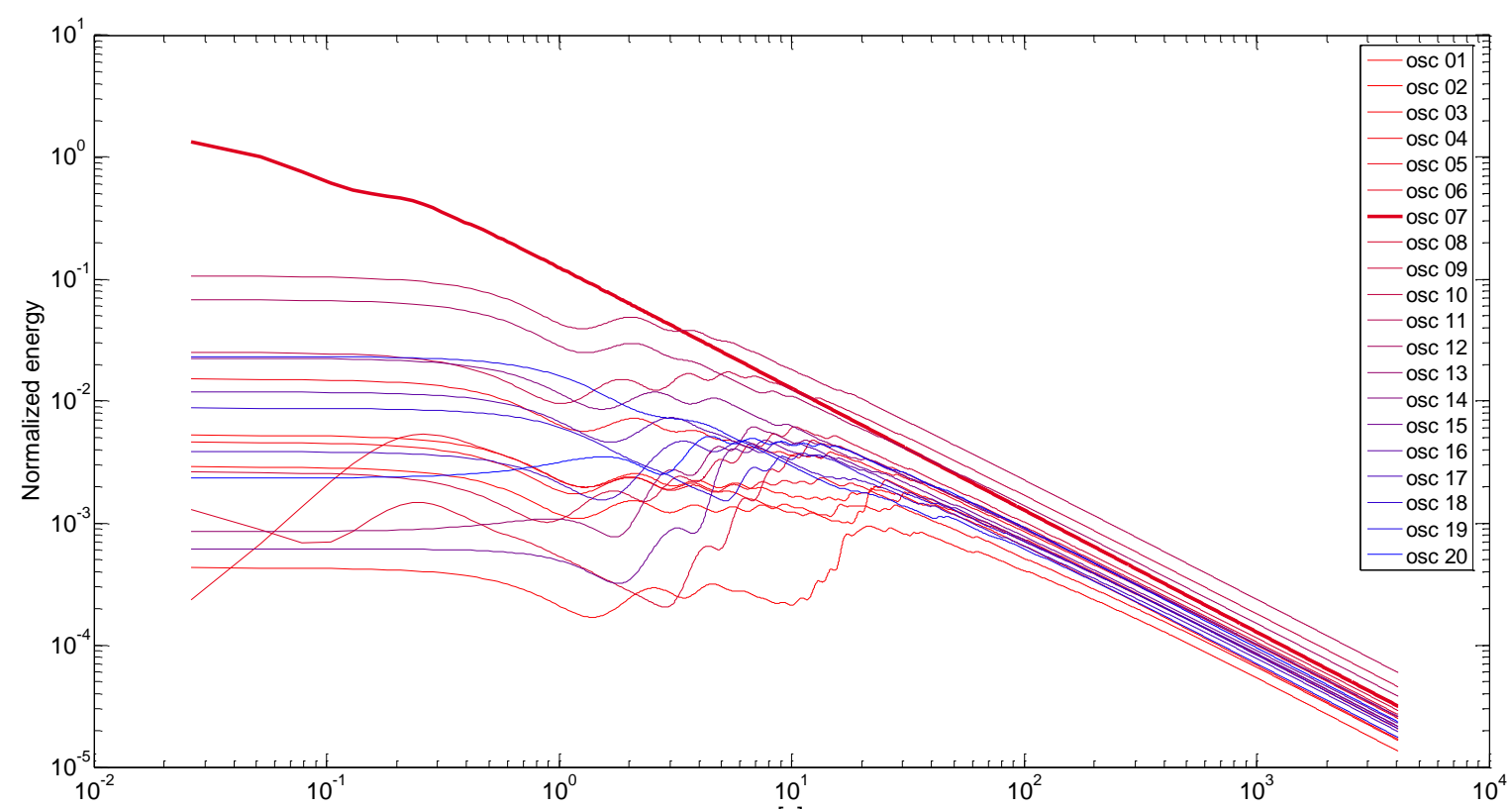

[s]

Fig. 15. Chain with mass focus with non-proportional damping, time energy averages versus time, $x, y$ axes in log scale.

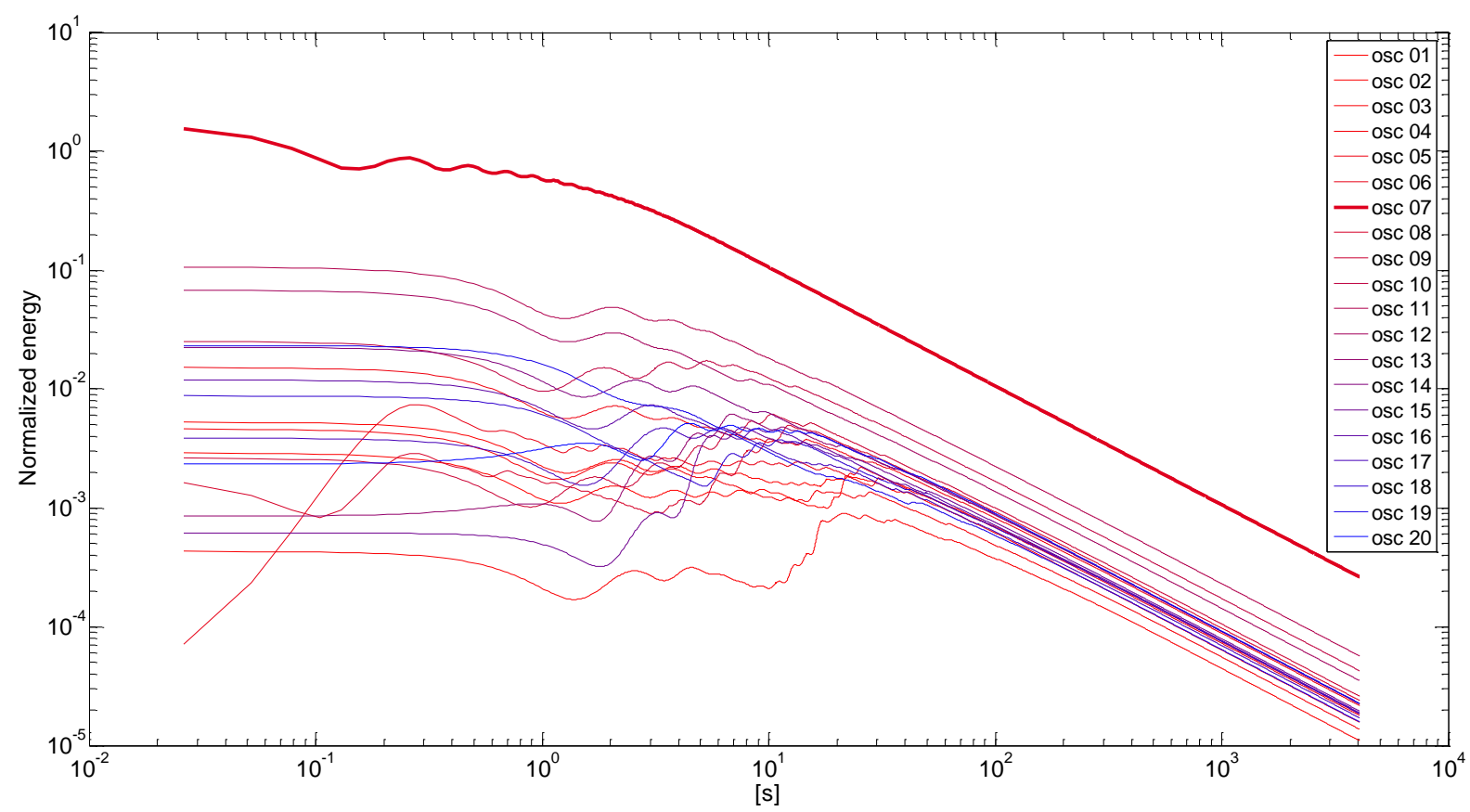

Fig. 16. Chain with mass focus with uniform nondimensional damping coefficient, time energy averages versus time, $x, y$ axes in log scale.

Even though damping diminishes the energy storage into the focus, Fig. 14 enlightens $\vartheta(t)$ remains well above 1, thus the energy focus is effective also for damped systems, as forecasted by Eq. (33).

The case of non-proportional damping affects the process of energy localization. Considering a uniform distribution of damping along the chain, Fig. 15 shows the moving averages of the chain of oscillators in the presence of the mass focus. The conclusions are: 


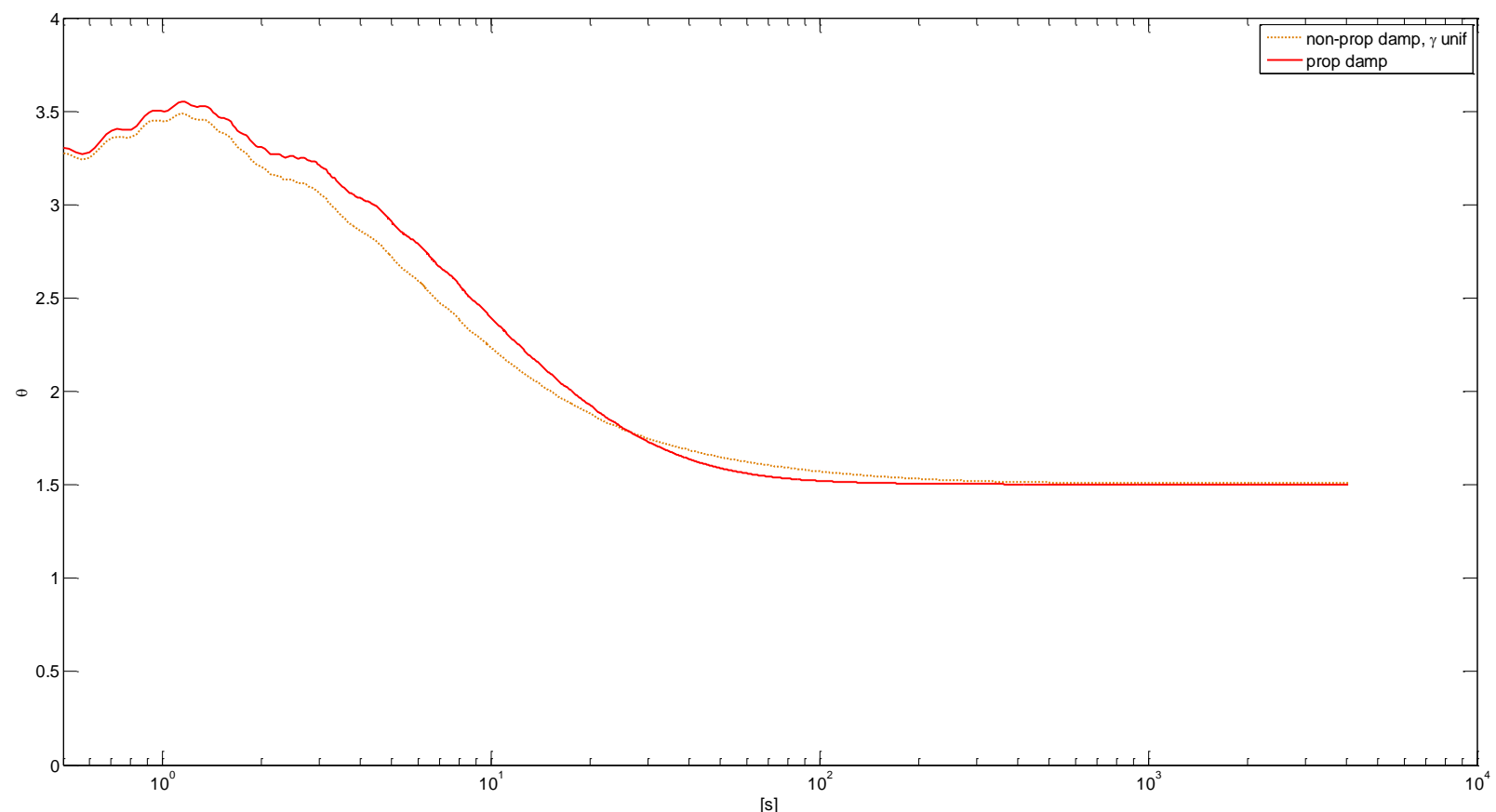

Fig. 17. Energy coupling index versus time, $x, y$ axes in log scale, for proportional and non-proportional damping, in solid and dashed lines, respectively.

- since $\gamma_{r} \gg \gamma$, the motion of the focus is over damped and the slope of the curve is much stepper than the others, as predicted by Eq. (27);

- the motion of the other resonators is qualitatively analogous to the one for proportional damping, shown in Fig. 13.

It follows that nearly the whole energy, initially injected into the focus, is quickly dissipated and not substantially transferred to the other resonators.

Finally, the case of a uniform nondimensional damping coefficient $\gamma$ is considered.

Figure 16 shows the moving averages of the system, where $\frac{c_{r}}{c}=\sqrt{\frac{m_{r}}{m}}=\frac{1}{10}$, and Fig. 17 shows the energy spreading indexes. As expected these curves are similar to those obtained for proportional damping.

\section{Conclusions}

This paper proposes an additional contribution in the context of energy analysis of structures, providing a demonstration of a general energy decomposition property. The outlined results help in understanding localization and equipartition of energy, and can be used as a design tool for a given category of structures. In fact, in many applications is of interest to protect some regions of the structure, as for example in aerospace launchers and satellite constructions, where special electronic instruments are installed on board. Here, a new mathematical formulation for this class of problems is given, through which is possible to design the structural system such as the injected shock energy is piloted and routed towards an energy focus within the structure. This permits to avoid uncontrolled energy propagation along the system, preventing damages in predefined safety regions of the structures, introducing one (or more) sacrificial energy storage. 


\section{References}

[1] R.H. Lyon, Statistical energy analysis of dynamical systems: Theory and applications, Journal of Sound and Vibration 182 (1975), 59-76.

[2] R.C. Tolman, The Principles of Statistical Mechanics, Oxford: Clarendon Press. Reissued, New York, 1979.

[3] R.S. Langley, A general derivation of the statistical energy analysis equations for coupled dynamic systems, Journal of Sound and Vibration 135 (1989), 499-508.

[4] J. Woodhouse, An approach to the theoretical background of statistical energy analysis applied to structural vibration, Journal of the Acoustical Society of America 69 (1981), 1695-1709.

[5] Le Bot, Derivation of statistical energy analysis from radiative exchanges, Journal of Sound and Vibration 300 (2007), 763-779.

[6] Y. Kishimoto and D.S. Berrnstein, Thermodynamic modelling of interconnected systems, I Conservative coupling, Journal of Sound and Vibration 182 (19956), 23-58.

[7] Y. Kishimoto and D.S. Berrnstein, Thermodynamic modelling of interconnected systems, II Dissipative coupling, Journal of Sound and Vibration 182 (1995), 59-76.

[8] Carcaterra, Ensemble energy average and energy flow relationships for nonstationary vibrating systems, Journal of Sound and Vibration 288(3) (2005), 751-790.

[9] W.M. Hassad, V. Chellaboina and S.G. Nersesov, Thermodynamics, Priceton University Press, Princeton Series in Applied Mathematics, 2005.

[10] W.M. Hassad, V. Chellaboina and S.G. Nersesov, Impulsive and Hybrid Dynamical Systems, Priceton University Press, Princeton Series in Applied Mathematics, 2006.

[11] F. Magionesi and A. Carcaterra, Insights into the energy equipartition principle in undamped engineering structures, Journal of Sound and Vibration 322(4,5) (2008), 851-869.

[12] N. Roveri, A. Carcaterra and A. Akay, Energy equipartition and frequency distribution in complex attachments, Journal of the Acoustical Society of America 126(1) (2009), 122-128.

[13] Carcaterra An entropy formulation for the analysis of energy flow between mechanical resonators, Mechanical Systems and Signal Processing 16 (2002), 905-920.

[14] Le Bot, Entropy in Statistical Energy Analysis, Journal of Acoustical Society of America 125 (2009), 1473-1478.

[15] Le Bot and A. Carcaterra, Mazur, Statistical Vibro-acoustics and Entropy Concept, Entropy Journal, 2011.

[16] D.S. Bernstein and S.P. Bath, Energy equipartition and the emergence of damping in lossless systems, Proc. IEEE Conf. Dec. Contr., 2002, pp. 2913-2918.

[17] A. Carcaterra, A. Akay and I.M. Koç, Nearly irreversible energy trapping by an undamped continuous structure with singularity points in its modal density, Journal of Acoustical Society of America 119 (2006), 2124.

[18] A. Carcaterra and A. Akay, Theoretical foundation of apparent damping and energy irreversible energy exchange in linear conservative dynamical systems, Journal of Acoustical Society of America 121 (2007), 1971-1982.

[19] A. Carcaterra and A. Akay, Dissipation in a finite-size bath, Physical Review E 84 (2011).

[20] S. De Rosa and F. Franco, A scaling procedure for the response of an isolated system with high modal overlap factor, Mechanical Systems and Signal Processing 22(7) (2008), 1549-1565.

[21] S. De Rosa and F. Franco, On the use of the asymptotic scaled modal analysis for time-harmonic structural analysis and for the prediction of coupling loss factors for similar systems, Mechanical Systems and Signal Processing 24(2) (2010).

[22] F. Giorgiadis, F. Vakakis, D.M. McFarland and L. Bergman, Shock isolation through passive energy pumping caused by non-smooth nonlinearities, International Journal of Bifurcation and Chaos 15(6) (2005), 1989-2001.

[23] A.F. Vakakis and O. Gendelman, Energy pumping in nonlinear mechanical oscillators II: Resonance capture, J Appl Mech 68 (2001), $42-48$.

[24] A.F. Vakakis, Shock isolation through the use of nonlinear energy sinks, J Vibr Contr 9 (2003) (Commemorative Issue on the Occasion of Professor Friedrich G. Pfeiffer's 65th Birthday), 79-93.

[25] A.F. Vakakis, L.I. Manevitch, O. Gendelman and L. Bergman, Dynamics of linear discrete systems connected to local essentially nonlinear attachments, J Sound Vibr 264 (2003), 559-577.

[26] A.F. Vakakis, O. Gendelman, L.A. Bergman, D.M. McFarland, G. Kerschen and Y.S. Lee, Nonlinear targeted energy transfer in mechanical and structural systems, Springer, 2008.

[27] F. dell'Isola and S. Vidoli, Damping of bending waves in truss beams by electrical transmission lines with PZT actuators, Archive of Applied Mechanics 68 (1998), 626-636.

[28] U. Andreaus, F. dell'Isola and M. Porfiri, Piezoelectric passive distributed controllers for beam flexural vibrations, Journal of Vibration and Control 10 (2004), 625.

[29] M. Porfiri, F. dell'Isola and F.M. Frattale Mascioli, Circuit analog of a beam and its application to multimodal vibration damping, using piezoelectric transducers, International Journal of Circuit Theory and Applications 32 (2004), 167-198.

[30] C. Maurini, J. Pouget and F. dell'Isola, On a model of layered piezoelectric beams including transverse stress effect, International Journal of Solids and Structures 41 (2004), 4473-4502.

[31] C. Maurini, F. dell'Isola and D. Del Vescovo, Comparison of piezoelectronic networks acting as distributed vibration absorbers, Mechanical Systems and Signal Processing 18 (2004), 1243-1271.

[32] F. dell'Isola and P. Seppecher, The relationship between edge contact forces, double forces and interstitial working allowed by the principle of virtual power, Comptes Rendus de l'Academie de Sciences - Serie IIb: Mecanique, Physique, Chimie, Astronomie 321 (1995), 303-308.

[33] F. dell'Isola and P. Seppecher, Edge contact forces and quasi-balanced ower, Meccanica 32 (1997), $33-52$.

[34] F. dell'Isola and A. Romano, On the derivation of thermomechanical balance equations for continuous systems with a nonmaterial interface, International Journal of Engineering Science 25 (1987), 1459-1468.

[35] F. dell'Isola and A. Romano, A phenomenological approach to phase transition in classical field theory, International Journal of Engineering Science 25 (1987), 1469-1475. 

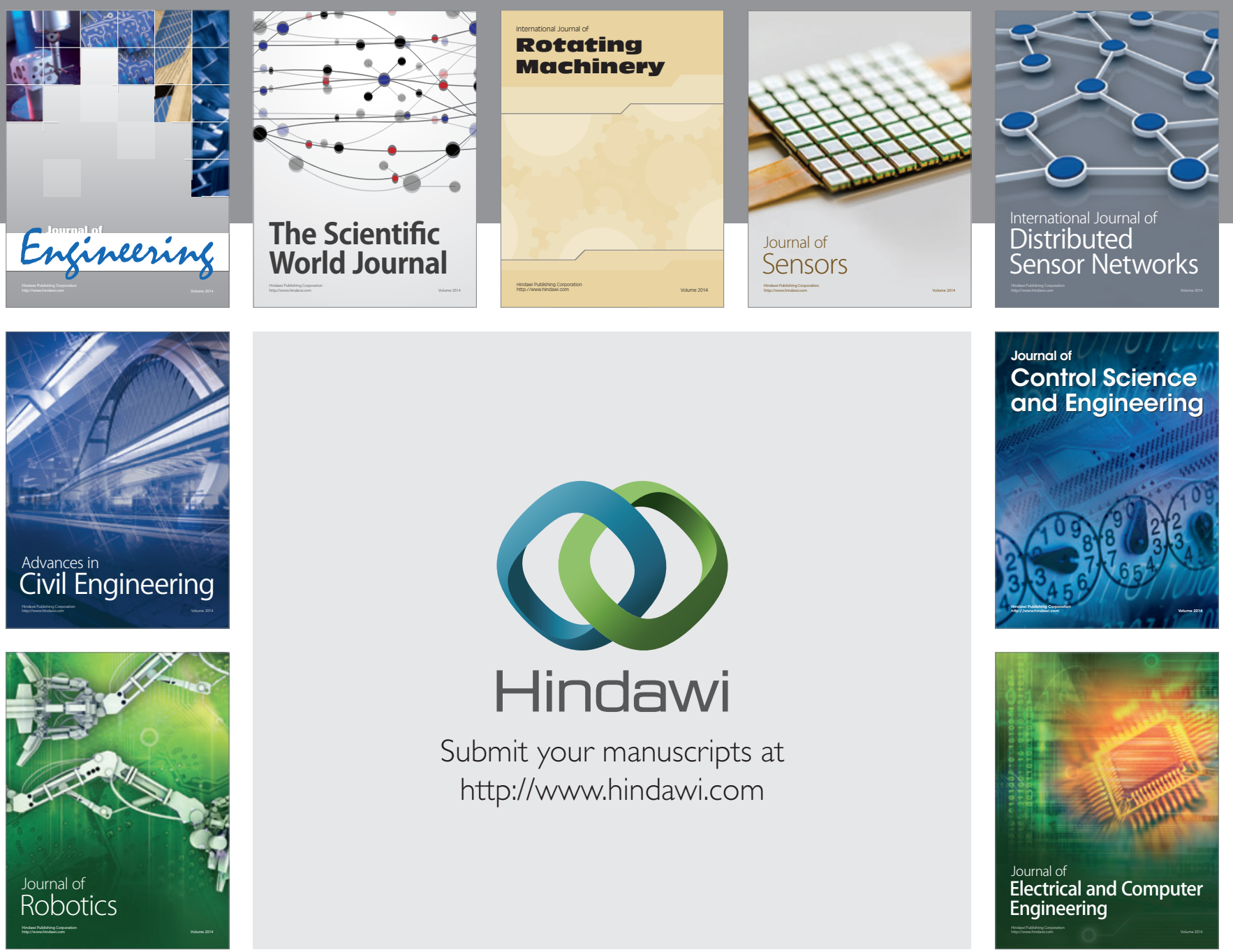

Submit your manuscripts at

http://www.hindawi.com
